\title{
Two light stops in the NMSSM and the LHC
}

\author{
Jyotiranjan Beuria, ${ }^{a, b}$ Arindam Chatterjee, ${ }^{a, b}$ AseshKrishna Datta ${ }^{a}$ \\ and Santosh Kumar Rai ${ }^{a, b}$ \\ ${ }^{a}$ Harish-Chandra Research Institute, \\ Allahabad 211019, India \\ ${ }^{b}$ Regional Centre for Accelerator-based Particle Physics, \\ Harish-Chandra Research Institute, Allahabad 211019, India \\ E-mail: jyotiranjan@hri.res.in, arindam@hri.res.in, asesh@hri.res.in, \\ skrai@hri.res.in
}

ABSTRACT: We study the viability of having two relatively light top squarks ('stops') in the framework of the Next-to-Minimal Supersymmetric Standard Model (NMSSM). Such light stops render the NMSSM rather 'natural'. These are shown to be allowed by the relevant direct searches at the Large Hadron Collider (LHC) and to be compatible with the latest LHC results on the Higgs sector, other low energy electroweak constraints and recent constraints from the dark matter (DM) sector. We propose dedicated searches for such light stops at the LHC within a 'simplified' scenario that may have a bino-like or a singlino-like neutralino LSP as the DM candidate and point out various final states carrying the imprint of their collective presence. Under certain circumstances, in such a scenario, presence of two light stops may give rise to final states which are not so typical in their search. Thorough studies at the detector level reveal the status of such a scenario after the $8 \mathrm{TeV}$ run of the LHC and shed light on the prospects of its 13 and $14 \mathrm{TeV}$ runs. In favorable regions of the NMSSM parameter space, with low-lying spectra, signals with significance $\gtrsim 5 \sigma$ are possible with a few tens to a few hundreds of $\mathrm{fb}^{-1}$ of integrated luminosity in diverse final states.

KEYWoRDs: Supersymmetry Phenomenology

ARXIV EPRINT: 1505.00604 


\section{Contents}

1 Introduction 1

2 The situation with two light stops in the NMSSM 3

2.1 The stop and the sbottom sectors of the NMSSM 4

2.2 The SM-like Higgs boson in the NMSSM 5

2.3 NMSSM versus MSSM: two light stops and the Higgs mass 6

2.4 The neutralino sector of the NMSSM 9

3 The compatible parameter space of the NMSSM 10

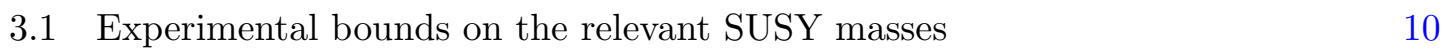

$\begin{array}{lll}3.1 .1 & \text { Scenario with a bino-like LSP } & 12\end{array}$

$\begin{array}{ll}\text { 3.1.2 Scenario with a singlino-like LSP } & 12\end{array}$

$\begin{array}{lll}3.2 & \text { Allowed regions of the NMSSM parameter space } & 13\end{array}$

$\begin{array}{lll}3.3 & \text { Choosing the benchmark scenarios } & 19\end{array}$

4 A pair of light stops at the LHC $\quad \mathbf{2 2}$

4.1 Cascade decay of the heavier stop and possible final states 22

$\begin{array}{ll}4.2 & \text { Signatures at the LHC } 26\end{array}$

$\begin{array}{lll}4.3 \text { Simulation } & 29\end{array}$

$\begin{array}{lll}\text { 4.3.1 Reconstructing the physics objects } & 30\end{array}$

$\begin{array}{lll}4.3 .2 & \text { Event selection } & 31\end{array}$

$\begin{array}{lll}\text { 4.3.3 Results and discussions } & 32\end{array}$

5 Conclusions $\quad 36$

\section{Introduction}

The observation of a scalar boson, now appearing more and more to be the Higgs boson, by the ATLAS and the CMS collaborations $[1,2]$ of the Large Hadron Collider (LHC), has accounted for the last missing piece in the particle spectrum of the Standard Model (SM) of particle physics and has vindicated the mechanism of the breaking of the electroweak symmetry as incorporated in the SM. Notwithstanding the fact that the SM can now safely be considered as an essential part of our understanding of the micro-world, there are ample reasons to believe that it does not offer the complete picture of what Nature has chosen for us. There are issues which are not understood by staying within the SM and require ideas extending the SM-picture of particle physics. This has led to a plethora of models beyond the SM (BSM) which one way or the other predict new exotic particle states in addition to the SM ones. The experimental results from the ATLAS and CMS experiments with 
center of mass energies of 7 and $8 \mathrm{TeV}$, have not only confirmed the predictions of the SM, including that of the Higgs boson, but have also started pushing the energy scale up for a possible BSM physics scenario.

Supersymmetry (SUSY) has been one of the most popular BSM scenarios and is rather thoroughly studied in the last three decades. Its simplest incarnation, the so-called minimal supersymmetric standard model (MSSM), offers a rich phenomenology involving the SUSY particles (sparticles, the partners of the SM excitations differing only in their spins) that would be present below the $\mathcal{O}(\mathrm{TeV})$ scale on the ground of naturalness and hence, supposed to be within the reach of the LHC experiments. However, no hint of such excitations has been found in the experiments to date and data from the LHC have already put rather stringent limits on the masses of such particles [3, 4]. In addition, the recent observation of a scalar resonance at $\sim 125 \mathrm{GeV}$ followed by the studies of its properties reveal an impressive agreement with the predictions of the SM. These have put the MSSM under tremendous scrutiny. Although a light CP-even scalar eigenstate with a mass of $125 \mathrm{GeV}$ is possible in the MSSM through radiative corrections, it prefers the third generation squark mass (in particular, the stop mass) to be much larger than $\mathcal{O}(1 \mathrm{TeV})$ and therefore puts the most natural solution to the so-called "gauge hierarchy problem" on somewhat troubled grounds by requiring an uncomfortable degree of fine-tuning.

Thus, within the MSSM, on the ground of naturalness, there is a palpable tension between the observed mass of the Higgs boson and the stop mass. This is a version of the so-called "little hierarchy problem" [5]. Such an issue can be ameliorated in an extension of the MSSM called the next-to-MSSM (NMSSM) [6] where the MSSM is augmented by an additional singlet scalar superfield. In such a scenario one can find, in contrast, tree level contributions to the Higgs masses that depend upon the new free parameters. Thus, the requirement of rather massive stops to obtain a $\sim 125 \mathrm{GeV}$ Higgs boson gets relaxed to a significant extent [7-11] and sub-TeV masses for both stops could easily serve the purpose. As far as the naturalness criterion is concerned, such a scenario conforms to the yardstick in a more efficient way than a scenario with only one relatively light stop [12]. However, the issue of "naturalness" is a more involved one and relates to other parameters in the theory in varied degrees. In the present study, we focus on such a scenario within the NMSSM where both stops are light (with sub-TeV masses).

On the other hand, the search for supersymmetric particles at the LHC has not only been quite elaborate but turns out to be quite exclusive as well in setting limits on the sparticle masses. The explicit constraints do however mostly restrict the strongly interacting sector which is expected due to their large production cross sections at a hadron collider like the LHC. Nonetheless, the third generation squarks present themselves with the weakest of the bounds from their direct searches. These happen mainly because of their smaller production cross sections when compared with the same for the squarks from the first two generations and also due to their possibly longer decay chains which in turn weaken the signal strengths. Thus, rather light (sub-TeV) squarks from the third generation in a scenario like the NMSSM steals the spotlight and invigorates the program of dedicated studies for them at the LHC.

The paper is organized as follows. In section 2 we collect the basic ingredients for our study where we present the situation with two light stops in the NMSSM in reference to the 
observed mass of the Higgs boson. We also discuss the neutralino sector of the NMSSM which is going to have a direct bearing in our present study. Section 3 is dedicated to finding the region of the NMSSM parameter space compatible with constraints originating from theoretical demands (like the ones arising by demanding absence of Landau poles in the evolutions of various new couplings), cosmological experiments shedding light on the dark matter (DM) sector, the experimental analyses in the Higgs sector and the direct SUSY searches at the LHC and other collider experiments. A thorough scan of the NMSSM parameter space is undertaken for the purpose and a few benchmark scenarios are chosen for simulation studies. A detector-level simulation at the LHC is presented in section 4 for the benchmark scenarios along with a detailed simulation of the important SM backgrounds. We then discuss the prospects of discovering such light stops at the imminent LHC runs with higher center of mass energies. In section 5 we conclude.

\section{The situation with two light stops in the NMSSM}

In addition to the superfields appearing in the MSSM, NMSSM contains a singlet $\hat{S}$. In the following discussion we will confine ourselves to the $Z^{3}$ invariant NMSSM, i.e. we will ignore linear and bilinear terms in $\hat{S}$, as well as, the $\mu$ term. The superpotential reads [6]

$$
\mathcal{W}=\left.\mathcal{W}_{\mathcal{M S S M}}\right|_{\mu=0}+\lambda \hat{S} \hat{H}_{u} \cdot \hat{H}_{d}+\frac{\kappa}{3} \hat{S}^{3}
$$

where, $\mathcal{W}_{\mathcal{M S S M}}$ is the superpotential for the MSSM, $\hat{H}_{u}$ and $\hat{H}_{d}$ denote the doublet Higgs superfields while $\hat{S}$ denotes the gauge singlet superfield. The corresponding soft supersymmetry-breaking terms are given by

$$
-\mathcal{L}_{\text {soft }}=-\left.\mathcal{L}_{\text {soft }}^{\mathrm{MSSM}}\right|_{B \mu=0}+m_{S}^{2}|S|^{2}+\lambda A_{\lambda} S H_{u} . H_{d}+\frac{1}{3} \kappa A_{\kappa} S^{3}+\text { h.c. }+\ldots
$$

where, $\mathcal{L}_{\text {soft }}^{\text {MSSM }}$ denotes the soft supersymmetry-breaking terms in the MSSM; $A_{\lambda}$ and $A_{\kappa}$ are the soft supersymmetry-breaking terms with the dimension of mass; $m_{S}^{2}$ is the soft supersymmetry-breaking mass-squared terms for $S$. During electroweak symmetry breaking (EWSB) the CP-even neutral components of $H_{u}, H_{d}$ and $S$, which are the scalar components of superfields $\hat{H}_{u}, \hat{H}_{d}$ and $\hat{S}$ respectively, assume vacuum expectation values (vevs) $v_{u}, v_{d}$ and $v_{S}$ respectively. Consequently, an effective $\mu$ term $\left(\mu_{\text {eff }}\right)$ is generated, which is given by $\mu_{\text {eff }}=\lambda v_{S}$. This provides an elegant solution to the well-known " $\mu$ problem" [13] that plagues the MSSM while the NMSSM was originally motivated over this virtue (see [6] and references therein). From our knowledge of the MSSM, where the Higgsino mass parameter $\mu$ plays a pivotal role in connecting the Higgs, the stop (and the bottom squark (sbottom)) and the electroweak gaugino/higgsino (electroweakino) sectors, it is not difficult to realize how NMSSM parameters could make their interplay even more involved. Studies in the framework of the NMSSM discuss the situations with the Higgs sector [14-20], the nature and role of the LSP [14, 21-32]. Among these, the more recent ones take into account the LHC-results on the Higgs boson and other relevant constraints. Also, in the recent past, issues over the light stops and the sbottoms at the LHC [33-35] in 
the context of the NMSSM and their implications of the $7 \mathrm{TeV}$ (LHC-7) and $8 \mathrm{TeV}$ (LHC8) runs of the LHC (in addition to a relatively light gluino) have been discussed in some detail [36].

In the following subsections, we outline some relevant features of the three sectors mentioned above in the NMSSM framework before moving on to explore their implications in the context of the present work.

\subsection{The stop and the sbottom sectors of the NMSSM}

An NMSSM spectrum with two light stops inevitably contains at least one relatively light sbottom state. In particular, if the lightest stop $\left(\tilde{t}_{1}\right)$ has a dominant left component, the presence of a comparably light sbottom which is mostly left-chiral is automatic. This follows from the fact that the soft SUSY breaking mass term for the state $\tilde{b}_{L}$ has the same origin $\left(m_{\tilde{Q}_{3}}\right)$ as that for the state $\tilde{t}_{L}$ and they differ only by the so-called $D$-term contributions which are relatively small and are model-independent for a given value of $\tan \beta$. The mass-squared matrices involving the $\tilde{t}$ (in the basis $\tilde{t}_{L}, \tilde{t}_{R}$ ) and $\tilde{b}$ (in the basis $\tilde{b}_{L}, \tilde{b}_{R}$ ) states in the NMSSM, (which, at tree-level, are similar to the ones in the case of MSSM) are given by [6]

$$
\mathcal{M}_{\tilde{t}}=\left(\begin{array}{cc}
m_{\tilde{Q}_{3}}^{2}+y_{t}^{2} v_{u}^{2}+\left(v_{u}^{2}-v_{d}^{2}\right)\left(\frac{g_{1}^{2}}{12}-\frac{g_{2}^{2}}{4}\right) & y_{t}\left(A_{t} v_{u}-\mu_{\mathrm{eff}} v_{d}\right) \\
y_{t}\left(A_{t} v_{u}-\mu_{\mathrm{eff}} v_{d}\right) & m_{\tilde{U}_{3}}^{2}+y_{t}^{2} v_{u}^{2}-\left(v_{u}^{2}-v_{d}^{2}\right) \frac{g_{1}^{2}}{3}
\end{array}\right)
$$

and

$$
\mathcal{M}_{\tilde{b}}=\left(\begin{array}{cc}
m_{\tilde{Q}_{3}}^{2}+y_{b}^{2} v_{d}^{2}+\left(v_{u}^{2}-v_{d}^{2}\right)\left(\frac{g_{1}^{2}}{12}+\frac{g_{2}^{2}}{4}\right) & y_{b}\left(A_{b} v_{d}-\mu_{\mathrm{eff}} v_{u}\right) \\
y_{b}\left(A_{b} v_{d}-\mu_{\mathrm{eff}} v_{u}\right) & m_{\tilde{D}_{3}}^{2}+y_{b}^{2} v_{d}^{2}+\left(v_{u}^{2}-v_{d}^{2}\right) \frac{g_{1}^{2}}{6}
\end{array}\right) .
$$

Note that $\mu_{\text {eff }}\left(=\lambda v_{S}\right)$ replaces $\mu$ in the off-diagonal terms of these mass-squared matrices when compared to the MSSM case. $m_{\tilde{Q}_{3}}$ stands for the soft SUSY breaking mass term for the third generation doublet (left-handed) squarks while $m_{\tilde{U}_{3}}$ and $m_{\tilde{D}_{3}}$ denote the same for the singlet (right-handed) stop and sbottom states, respectively. $A_{i}$-s are the trilinear soft SUSY breaking terms and $y_{i}$-s denote the respective Yukawa couplings. $v_{u}$ and $v_{d}$ represent the vevs of the CP-even up- and down-type neutral Higgs bosons, $H_{u}^{0}$ and $H_{d}^{0}$, respectively and $g_{2}$ and $g_{1}$ denote the gauge couplings corresponding to $\mathrm{SU}(2)_{L}$ and $\mathrm{U}(1)_{Y}$ gauge groups, respectively. Smaller values of $m_{\tilde{Q}_{3}}$ and $m_{\tilde{U}_{3}}$ ensure lightness of both stops. Further, as has been explained earlier, small $m_{\tilde{Q}_{3}}$ corresponds to one light sbottom (which is dominantly left-handed). Proximity of $m_{\tilde{Q}_{3}}$ and $m_{\tilde{U}_{3}}$, and the largeness of $y_{t}$, guarantee significant mixing between the left- and the right-handed stops. As a result, there may be a good amount of splitting between the two stop mass-eigenstates. This would then favor a hierarchy of low-lying stop and sbottom masses of the following kind: $m_{\tilde{t}_{1}}<m_{\tilde{b}_{1}}<m_{\tilde{t}_{2}}$, which we study in some detail in the later part of this work. ${ }^{1}$ The chiral admixtures of the individual states depend on the hierarchy of the soft masses $m_{\tilde{Q}_{3}}, m_{\tilde{U}_{3}}$ and $m_{\tilde{D}_{3}}$.

\footnotetext{
${ }^{1}$ Hierarchies like $m_{\tilde{b}_{1}} \lesssim m_{\tilde{t}_{1}} \lesssim m_{\tilde{t}_{2}}$ and $m_{\tilde{t}_{1}} \lesssim m_{\tilde{t}_{2}} \lesssim m_{\tilde{b}_{1}}$ are also possible depending upon the mutual hierarchy of $m_{\tilde{Q}_{3}}$ and $m_{\tilde{U}_{3}}$.
} 


\subsection{The SM-like Higgs boson in the NMSSM}

In the NMSSM, on EWSB, there are three CP-even Higgs bosons which are mixtures of CP-even (real) parts of $H_{u}, H_{d}$ and $S$. The LHC results now require one of these to be SM-like [37]. The mass $\left(m_{h}\right)$ of the SM-like Higgs boson $(h)$ is given by [38]

$$
m_{h}^{2}=m_{Z}^{2} \cos ^{2} 2 \beta+\lambda^{2} v^{2} \sin ^{2} 2 \beta+\Delta_{\text {mix }}+\Delta_{\text {rad.corr. }}
$$

where $v=\sqrt{v_{u}^{2}+v_{d}^{2}} \simeq 174 \mathrm{GeV}, \beta=\tan ^{-1} \frac{v_{u}}{v_{d}}$. The first term on the right hand side of this equation stands for the tree level squared mass of the Higgs boson in the MSSM. The second term is the NMSSM contribution at the tree level. The third term stems from singlet-doublet mixing which, in the limit of weak mixing, is given by

$$
\Delta_{\text {mix }}=\frac{4 \lambda^{2} v_{S}^{2} v^{2}(\lambda-\kappa \sin 2 \beta)^{2}}{\tilde{m}_{h}^{2}-m_{s s}^{2}}
$$

where $\tilde{m}_{h}^{2}=m_{h}^{2}-\Delta_{\text {mix }}$ and $m_{s s}^{2}=\kappa v_{S}\left(A_{\kappa}+4 \kappa v_{S}\right)$. As explored in reference [38], it is possible to raise the Higgs mass up to $140 \mathrm{GeV}$ assuming perturbativity of $\lambda$ up to the GUT scale (i.e., no Landau pole is developed in the evolution of $\lambda$ ). If one is ready to sacrifice such a requirement (the plausibility of which has recently been discussed in the framework of a scenario like $\lambda$ SUSY), it is possible to have the doublet-like Higgs boson as heavy as $\sim 300 \mathrm{GeV}$ [38-40]. Thus, even with a relatively small contribution from $\Delta_{\text {rad.corr. }}$, the second and the third term could raise the tree-level Higgs mass significantly. However, note that $\Delta_{\text {mix }}$ could have either sign and can increase or reduce the doublet-like Higgs mass.

On the contrary, in the MSSM, the lightest CP-even Higgs boson mass is bounded from above by $m_{Z} \cos 2 \beta$ at tree-level. Hence, in such a scenario, a Higgs boson with mass $\sim 125 \mathrm{GeV}$, as observed by the LHC experiments, requires a significant amount of radiative correction $\left(\Delta_{\text {rad.corr. }}\right)$ which mostly arises at one-loop level with top quark and the stops in the loops thanks to the large top Yukawa coupling [41-43]. For a fixed $\tan \beta$, with $m_{A} \gg m_{Z}$, the SM-like light CP-even Higgs mass [up to $\mathcal{O}\left(\frac{m_{Z}^{2}}{m_{A}^{2}}\right)$ ] is given by [47]

$$
m_{h}=\sqrt{\left(m_{Z}^{2} \cos ^{2} 2 \beta+\epsilon \sin ^{2} \beta\right)}\left[1+\frac{\epsilon m_{Z}^{2} \cos ^{2} \beta}{2 m_{A}^{2}\left(m_{Z}^{2}+\epsilon \sin ^{2} \beta\right)}-\frac{m_{Z}^{2} \sin ^{2} \beta+\epsilon \cos ^{2} \beta}{2 m_{A}^{2}}\right]
$$

where $m_{A}$ is the soft SUSY-breaking mass for the pseudoscalar Higgs boson and the oneloop correction $\epsilon \simeq \Delta_{\text {rad.corr. }}$ is given by [44-47]

$$
\epsilon=\frac{3 m_{t}^{4}}{4 \pi^{2} v^{2} \sin ^{2} \beta}\left[2 \log \frac{M_{S}}{m_{t}}+\frac{X_{t}^{2}}{M_{S}^{2}}\left(1-\frac{X_{t}^{2}}{12 M_{S}^{2}}\right)\right] .
$$

In the above expression, $m_{t}$ denotes the mass of the top quark, ${ }^{2} M_{S}=\sqrt{m_{\tilde{t}_{1}} m_{\tilde{t}_{2}}}$ and $X_{t}=A_{t}-\mu \cot \beta$. It turns out that at least one of the $\tilde{t}$ states is required to be heavier than about a $\mathrm{TeV}$ and a large mixing between $\tilde{t}_{L}$ and $\tilde{t}_{R}$ is needed to push the (light) Higgs mass up to its experimentally observed value [48-53].

\footnotetext{
${ }^{2}$ To take into account the leading two-loop corrections to the Higgs mass, the running $\overline{M S}$ top quark mass is to be taken [47].
} 
As evident from the discussion above, in the NMSSM, in addition to the MSSM contribution, the Higgs mass receives extra contribution at the tree level as shown in equation (2.5). Thus, one does not necessarily bank on a heavy $\tilde{t}$ and/or large mixing to obtain a heavier Higgs boson [39, 40, 54] with its mass around $\sim 125 \mathrm{GeV}[15,16,55,56]$ as observed by the LHC experiments. This opens up, in the present context, the hitherto unexplored possibility of a theoretically consistent NMSSM scenario where both $\tilde{t}_{1}$ and $\tilde{t}_{2}$ have sub-TeV masses and can be quite light. ${ }^{3}$ Such a provision warrants a thorough understanding of its implications, in particular, once it could survive the current bounds on stop masses from the LHC experiments. In the following subsection we highlight the compatibility of having two relatively light stops in the NMSSM. We also take a close look at its immediate implications for the scenario and contrast them with the situation in the MSSM.

\subsection{NMSSM versus MSSM: two light stops and the Higgs mass}

To demonstrate how light the two stops could get to be, we scan over the NMSSM parameter space with the following ranges for various input parameters using the popular package NMSSMTools (v4.4.0) [58-60, 62, 63]:

$$
\begin{gathered}
0.40 \leq \lambda \leq 0.75, \quad 0.01 \leq \kappa \leq 0.75, \quad\left|\mu_{\mathrm{eff}}\right| \leq 1 \mathrm{TeV}, \quad 1 \leq \tan \beta \leq 5, \\
\left|A_{\lambda}\right| \leq 2 \mathrm{TeV}, \quad\left|A_{\kappa}\right| \leq 2 \mathrm{TeV}, \quad\left|A_{t}\right| \leq \mathbf{3} \mathrm{TeV}, \\
250 \mathrm{GeV} \leq\left(m_{\tilde{Q}_{3}}, m_{\tilde{U}_{3}}\right) \leq 3 \mathrm{TeV} .
\end{gathered}
$$

The range of $\lambda$ ensures that we work in a large $\lambda$ regime for which the tree-level NMSSM contribution to the SM-like Higgs mass is appreciable. The range of Higgs mass that we allow in the scan is $125.1 \pm 3.0 \mathrm{GeV} .{ }^{4}$ As can be seen from equation (2.5), the tree-level NMSSM contribution to the Higgs mass is significant for small $\tan \beta$. Hence our choice for the range of the same in equation (2.9).

In figure 1 we compare the NMSSM (in blue) and the MSSM (in red) in the $m_{\tilde{t}_{1}}-m_{\tilde{t}_{2}}$ mass plane by scanning over the respective parameter spaces. For the MSSM, the results we present are by using FeynHiggs (v2.10.3) [67-71]. However, we independently checked the same by running the popular spectrum generator SuSpect (v2.43) [72]. We find close agreement between the two at the level of scanned output, except for some minor deviations arising out of known shifts in the SM-like Higgs mass by $2-3 \mathrm{GeV}$ from the two packages, for any given parameter-set. We also find very good agreements with the

\footnotetext{
${ }^{3}$ Some such generic possibilities had earlier been discussed [12] in the context of 'natural' SUSY [57] without trying to address the observed Higgs mass within the SUSY framework (thus implicitly relying on an NMSSM-like setup for providing the extra contribution to the Higgs mass).

${ }^{4}$ Note that the experimental uncertainty in determining $m_{h}$ is rather negligible $(\sim 240 \mathrm{MeV}[65])$. The uncertainty of $\pm 3 \mathrm{GeV}$ takes into account the theoretical uncertainty arising from missing higher order corrections in the MSSM (see, for example, reference [64] and references therein). Additional corrections to the Higgs mass proportional to the parameter $\lambda$ at 2-loop have been calculated in reference [66]. The uncertainty interval can even be a little higher if one considers the uncertainty in the measurement of the top quark mass.
} 


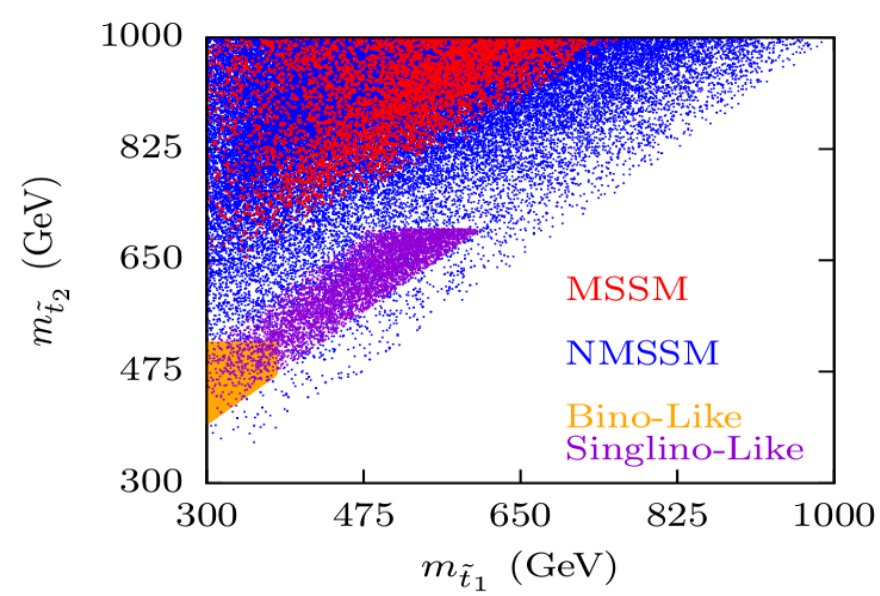

Figure 1. Scatter plot showing the allowed regions in the $m_{\tilde{t}_{1}}-m_{\tilde{t}_{2}}$ plane in the MSSM (in red; using FeynHiggs) and in the NMSSM (in blue; using NMSSMTools). Patches with yellow and purple points present regions in the NMSSM parameter space under specific scenarios (bino- and singlinodominated LSP neutralino, respectively) and satisfying some imposed spectral constraints discussed in section 3.2. For the ranges of the model parameters scanned over in the respective scenarios, see text.

existing literature $[49,50]$. The ranges of various MSSM parameters employed in the scan are as follows:

$$
\begin{gathered}
|\mu| \leq 2.5 \mathrm{TeV}, \quad 2 \leq \tan \beta \leq 32, \quad\left|A_{t}\right| \leq \mathbf{3} \mathrm{TeV} \\
200 \mathrm{GeV} \leq\left(m_{\tilde{Q}_{3}}, m_{\tilde{U}_{3}}, m_{A}\right) \leq 2.2 \mathrm{TeV}
\end{gathered}
$$

For both the NMSSM and the MSSM scans, we fix $M_{1}=300 \mathrm{GeV}, M_{2}=1.2 \mathrm{TeV}$ and $M_{3}=3 \mathrm{TeV}$. The soft masses for all other squarks and sleptons are fixed at $3 \mathrm{TeV}$. We set the corresponding trilinear parameters $A_{f}$ to zero and the top quark mass $m_{t}$ to $173.1 \mathrm{GeV}$. The edge along the diagonal traced out by the blue points marks the line below which $m_{\tilde{t}_{2}}<m_{\tilde{t}_{1}}$ which confronts their definitions.

It is clear from figure 1 , for $m_{\tilde{t}_{1}}$ as light as $300 \mathrm{GeV}$, the smallest $m_{\tilde{t}_{2}}$ that one can barely have in the MSSM is $\approx 650 \mathrm{GeV}$. In contrast, in the NMSSM, $\tilde{t}_{2}$ can be as light as $\approx 350 \mathrm{GeV}$. In fact, the figure also reveals that a near-degenerate pair of stops is not impossible in the NMSSM, being still consistent with the constraint from the Higgs mass. Over the entire range of mass shown in the figure, for any given $m_{\tilde{t}_{1}}$, NMSSM could provide us with a $\tilde{t}_{2}$ which can be lighter by $\approx 250-300 \mathrm{GeV}$ when compared to the MSSM.

In figure 2 we contrast the allowed regions in the $\frac{X_{t}}{M_{S}}$ plane for the MSSM (left) and the NMSSM (right). As shown, the color-code indicates the values of $\tan \beta$ (as is customary for the MSSM case) and $\lambda$ in the respective cases. It is evident from the left plot that in the MSSM, compatibility with observed mass of the Higgs boson requires either high values of $M_{S}$ or, in case of small $M_{S}$ (in turn, a small $m_{\tilde{t}_{1}}$ ), rather large $\frac{X_{t}}{M_{S}}$ (i.e., a large mixing parameter). Post Higgs discovery, this fact has become quite well-known and wellunderstood. Note that in the present work, we are interested in a pair of relatively light stops thus implying low $M_{S}$. For example, $m_{\tilde{t}_{1}} \approx 300 \mathrm{GeV}$ with $m_{\tilde{t}_{2}} \approx 500 \mathrm{GeV}$ results 

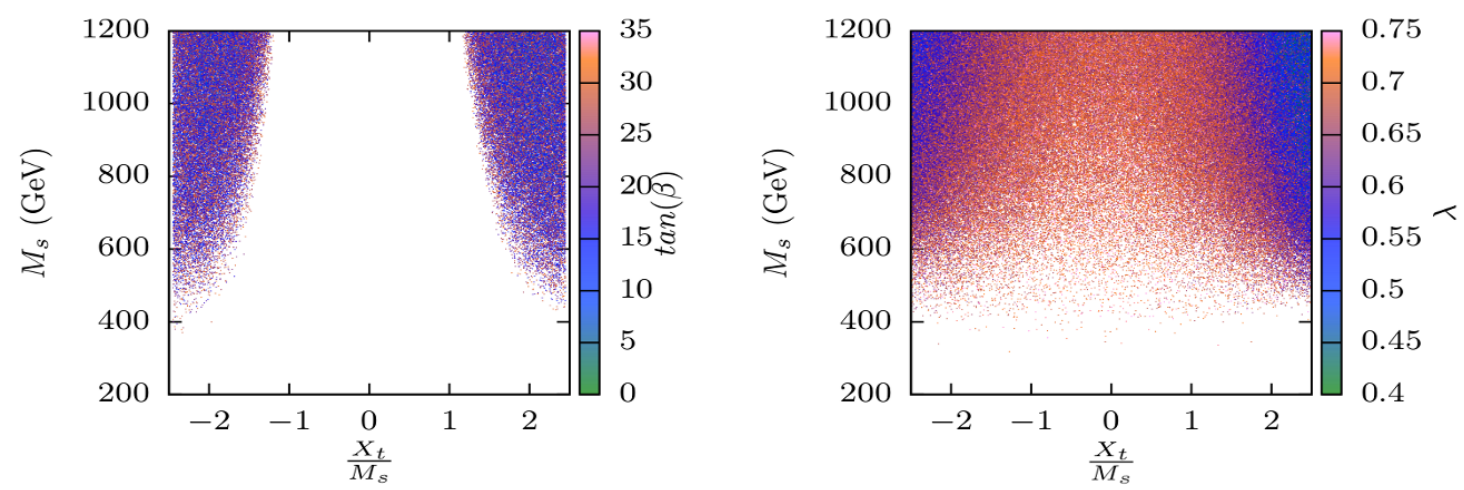

Figure 2. Scatter plots showing the regions in the $\frac{X_{t}}{M_{S}}-M_{S}$ plane compatible with the allowed range of the Higgs mass in the MSSM (left; using FeynHiggs) and in the NMSSM (right; using NMSSMTools). The color-code in the palette indicates the values of $\tan \beta$ and $\lambda$ for the left and the right plots, respectively. The range of parameters scanned over in the respective scenarios are same as in the case of figure 1 . See text for details.

in $M_{S} \approx 400 \mathrm{GeV}$. It is clear from figure 2 that the MSSM could hardly afford such a spectrum, and if at all, it would require a dangerously large value of $\frac{X_{t}}{M_{S}}(\approx \pm \sqrt{6}$, that corresponds to the so-called "maximal mixing scenario", and is the range considered in figure 2) that could lead to a charge and color-breaking minimum [73-77] for the scalar potential of the theory or an unstable electroweak vacuum [78, 79]. Furthermore, even for larger values of $M_{S}$, the required $\frac{X_{t}}{M_{S}}$ remains moderately large in the MSSM resulting in two well-separated allowed branches along the $\frac{X_{t}}{M_{S}}$ axis.

In contrast, from the plot in the right, we find that in the NMSSM, the 'desert' in the middle gets efficiently populated. This is since, in this region, Higgs mass gets significant tree level contribution in the NMSSM with $\lambda$ close to its largest value. Thus, unlike in the MSSM, a vanishing mixing parameter $\left(X_{t}\right)$ in the NMSSM is very much compatible with the observed mass of the Higgs boson. However, in a scenario where both stops are relatively light and hence the same for the soft masses for $\tilde{t}_{L}$ and $\tilde{t}_{R}$, it is not automatic that a small $X_{t}$ would correspond to a small mixing angle $\theta_{\tilde{t}}$ in the stop sector. This is because in the limit of vanishing mixing parameter the maximal mixing is still guaranteed if the diagonal terms of the $(2 \times 2)$ mass-squared matrix are equal. Given that we are interested in a scenario with two relatively light stops, the diagonal entries could be of comparable size. Thus, even with a vanishing $X_{t}$, a moderate mixing in the stop sector is possible.

In figure 3 we demonstrate this issue. Here we recast the right plot of figure 2 to indicate the amount of mixing, $\theta_{\tilde{t}}$, present in the stop sector. We observe, that there is a preponderance of states with moderate to small mixings (in green and blue) for small $X_{t}$, with only a few isolated cases having relatively large mixing (in purple and red). This is in sharp contrast to the MSSM scenario: in the NMSSM, it is possible to have a pair of relatively light stops and that also with low mixing. 


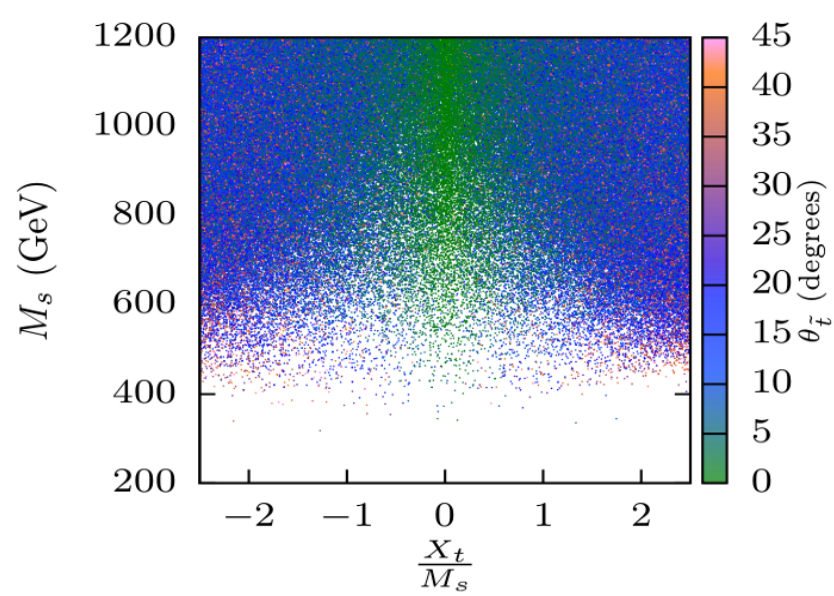

Figure 3. Same as in the right plot of figure 2 but with a palette indicating the mixing angle $\left(\theta_{\tilde{t}}\right.$; in degrees) in the stop sector.

\subsection{The neutralino sector of the NMSSM}

As in the case of MSSM, phenomenology of such light stop/sbottom squarks of the NMSSM depends on the electroweak gaugino sector in an essential way. In particular, the neutralino sector of the NMSSM differs from that of the MSSM in a crucial way. The fermionic component of the singlet superfield $\hat{S}$ in equation (2.1) (the 'singlino', $\widetilde{S}$ ) could mix with the gauginos and the higgsinos of the MSSM. A singlino-dominated neutralino could turn out to be the lightest supersymmetric particle (LSP) and may crucially affect the cascade decays of the heavier SUSY excitations [14,21-28]. We would further assume that $R$-parity is conserved and hence the $\operatorname{LSP}\left(\tilde{\chi}_{1}^{0}\right)$ is stable.

The symmetric $5 \times 5$ neutralino mass matrix (in the basis $\left\{\tilde{B}, \tilde{W}, \tilde{H}_{1}, \tilde{H}_{2}, \tilde{S}\right\}$ ) is given by

$$
\mathcal{M}_{0}=\left(\begin{array}{ccccc}
M_{1} & 0 & -\frac{g_{1} v_{d}}{\sqrt{2}} & \frac{g_{1} v_{u}}{\sqrt{2}} & 0 \\
& M_{2} & \frac{g_{2} v_{d}}{\sqrt{2}} & -\frac{g_{2} v_{u}}{\sqrt{2}} & 0 \\
& & 0 & -\mu_{\mathrm{eff}} & -\lambda v_{u} \\
& & & 0 & -\lambda v_{d} \\
& & & & \\
& & & & \\
& & & &
\end{array}\right),
$$

where $M_{1}$ and $M_{2}$ denote the soft SUSY-breaking masses for the U(1) $(\widetilde{B})$ and the $\mathrm{SU}(2)$ $(\widetilde{W})$ gauginos, respectively. All other variables are described earlier in the text. Note that the $(1,5)$ and the $(2,5)$ elements of the mass matrix are zeros. These imply that the singlino state does not mix directly with the $\widetilde{B}$ and the $\widetilde{W}$ states. Rather, its mixing to these states takes place indirectly via the higgsino sector. It is thus natural to expect that gauginosinglino mixings would never be too large. We consider two phenomenologically distinctive limiting possibilities with the neutralino LSP: (i) a bino-like LSP and (ii) a singlino-like one. Such possibilities, along with the choice of a minimal (simplified) scenario appropriate for 
the purpose, lead us to some benchmark scenarios. These are discussed in the next section. We do not discuss the case of a higgsino-like LSP in this work. This is since, as elaborated in the next section, we like to keep the charginos out from the phenomenology we discuss while a higgsino-like LSP would necessarily result in a light chargino. We postpone the discussion of such a possibility as well as the one that addresses a general situation of mixed electroweakinos.

\section{The compatible parameter space of the NMSSM}

The paradigm we are interested in is a 'simplified' but an eminently plausible scenario within the $Z_{3}$-symmetric NMSSM framework which is consistent with the latest Higgs data and satisfies other important experimental constraints. Essentially, we have two relatively light stops, one light sbottom and a neutralino-LSP which can be either bino- or singlinolike. We explore ranges where the lighter stop $\left(\tilde{t}_{1}\right)$ has mass below $\sim 550 \mathrm{GeV}$ while the heavier stop is not heavier than, say, $700 \mathrm{GeV}$. The lighter sbottom can have a mass in between the two stop masses. Such a spectrum of squarks from the third generation have moderate to significant pair-production cross sections at the imminent run of the LHC with increased center of mass energy and thus, is expected to be within its easy reach. Note that (as mentioned in the previous section) the lighter chargino $\left(\tilde{\chi}_{1}^{ \pm}\right)$is taken to be rather heavy with $m_{\tilde{\chi}_{1}^{ \pm}}>m_{\tilde{t}_{2}}$.

While exploring a spectrum with excitations as light as the ones mentioned above, it is imperative that one takes a critical note of relevant recent analyses reported by LHC-7 and LHC-8. Indeed, all four light SUSY excitations indicated above draw crucial bounds from the latest data. We first discuss these bounds which lead us to a few benchmark scenarios appropriate for our present study.

\subsection{Experimental bounds on the relevant SUSY masses}

Current experimental bounds from the LHC pertaining to the third generations squarks are grossly model-dependent in the sense that they refer to specific mass hierarchies among the involved states. It is thus important to check which of these bounds indeed apply for a scenario under study.

In the simplified scenario we consider, the heavier stop $\left(\tilde{t}_{2}\right)$ is the heaviest of the four relatively light SUSY states mentioned above. We assume $m_{\tilde{t}_{2}} \sim m_{t}+m_{\tilde{\chi}_{1}^{0}}$ such that the decay $\tilde{t}_{2} \rightarrow t \tilde{\chi}_{1}^{0}$ is suppressed. Such a requirement is only relevant when the LSP is binodominated while for a singlino-like LSP such a decay is naturally disfavored. Thus, $\tilde{t}_{2}$ would have two dominant decay modes: $\tilde{t}_{2} \rightarrow \tilde{t}_{1} Z$ and $\tilde{t}_{2} \rightarrow \tilde{b}_{1} W^{+} / W^{+*}{ }^{5}$ It is also possible that $\tilde{t}_{2}$ decays to $\tilde{t}_{1}$ and a light Higgs boson when kinematically allowed. By restricting the mass-split between $\tilde{t}_{2}$ and $\tilde{\chi}_{1}^{0}$ in the above fashion, we end up with somewhat compressed spectra comprising of closely spaced states like $\tilde{b}_{1}, \tilde{t}_{1}$ and the LSP below the state $\tilde{t}_{2}$.

\footnotetext{
${ }^{5}$ A stringent requirement of $m_{\tilde{t}_{1}}+m_{Z} \leq m_{\tilde{t}_{2}} \leq m_{\text {top }}+m_{\tilde{\chi}_{1}^{0}}$ would imply $m_{\tilde{t}_{1}}-m_{\tilde{\chi}_{1}^{0}}<m_{\text {top }}-m_{Z}(\approx$ $82 \mathrm{GeV}$ ) which is smaller than $m_{b}+m_{W}$. This would ensure $\tilde{t}_{1}$ always have a flavor violating $(\mathrm{FV})$ decay to $c \tilde{\chi}_{1}^{0}$ and/or four-body (4B) decay to $b f \bar{f}^{\prime} \tilde{\chi}_{1}^{0}$.
} 


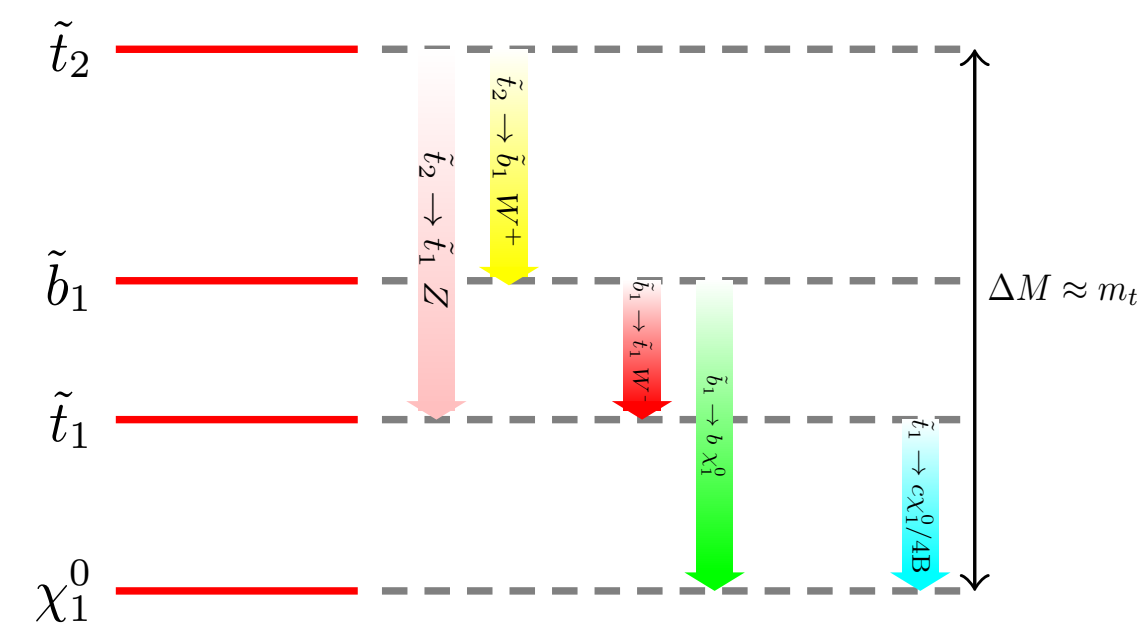

Figure 4. The mass-hierarchy of the lowest lying excitations that include two light stops and a light sbottom. The LSP $\tilde{\chi}_{1}^{0}$ can either be a bino or a singlino. The possible decay modes of these states are also shown. The mass-split between $\tilde{t}_{2}$ and $\tilde{\chi}_{1}^{0}(\Delta M)$ is required to be around the mass of the top quark for the simplified scenario discussed in this work. Note that the relative splittings shown in the figure are only representative and are not to scale.

As for $\tilde{b}_{1}$, in general, the two-body decay modes $\tilde{b}_{1} \rightarrow b \tilde{\chi}_{1}^{0}$ and $\tilde{b}_{1} \rightarrow \tilde{t}_{1} W^{-}$may both be substantial if kinematically allowed. However, if the LSP is singlino-dominated, the coupling $b \tilde{b}_{1} \tilde{\chi}_{1}^{0}$ will be suppressed and the decay $\tilde{b}_{1} \rightarrow \tilde{t}_{1} W^{-}$would prevail. Nonetheless, given that the mass-split between the states $\tilde{b}_{1}$ and $\tilde{t}_{1}$ may be naturally small in some regions of the SUSY parameter space, the latter mode (via on-shell or off-shell $W$-boson, as the case may be) might also experience a phase-space suppression resulting in a competition between these two available modes, as long as the singlino admixture in the LSP is not too large.

The lighter stop, in our scenario, could only undergo the loop-level flavor-violating decay $\tilde{t}_{1} \rightarrow c \tilde{\chi}_{1}^{0}[80]^{6}$ and/or a decay to a four-body final state $\tilde{t}_{1} \rightarrow b f \bar{f}^{\prime} \tilde{\chi}_{1}^{0}[82,83]$ (as $m_{\tilde{t}_{1}}-m_{\tilde{\chi}_{1}^{0}}<m_{b}+m_{W}$ ). Thus, bounds from only those experimental analyses that considered these possibilities would be relevant for the present study. We now briefly discuss the current experimental situation below.

Interestingly, direct production of a pair of $\tilde{t}_{2}$ and their decays to $\tilde{t}_{1} Z$ have been discussed for the first time by both the ATLAS and the CMS collaborations [84, 85] only very recently. The latter, in addition, considers the decay mode $\tilde{t}_{2} \rightarrow \tilde{t}_{1} h$. A phenomenological analysis of such a decay has been performed in reference [86]. It is to be noted that the decay mode $\tilde{t}_{2} \rightarrow \tilde{b}_{1} W^{+}$which is relevant for the scenario we are discussing in this work, has not yet been considered in the experimental studies to the best of our knowledge. In any case, the analyses mentioned in these works assume that $\mathrm{BR}\left[\tilde{t}_{1} \rightarrow t \tilde{\chi}_{1}^{0}\right]$ is $100 \%$

\footnotetext{
${ }^{6} \mathrm{~A}$ more recent work performs the complete one-loop calculation of the decay $\tilde{t}_{1} \rightarrow c \tilde{\chi}_{1}^{0}[81]$ in the framework of minimal flavor violation $(\mathrm{MFV})$. It is reported that a deviation of about $10 \%$ is typical for large values of the MFV scale while the same could be bigger if the MFV scale is small. We use NMSSMTools for our study which only has the results of reference [80] implemented.
} 


\begin{tabular}{|c|c|c|}
\hline Decay modes & ATLAS & CMS \\
\hline$\tilde{t}_{2} \rightarrow \tilde{t}_{1} Z$ & {$[84]$} & {$[85]$} \\
$\tilde{t}_{2} \rightarrow \tilde{t}_{1} h$ & Not available & {$[85]$} \\
$\tilde{t}_{2} \rightarrow \tilde{b}_{1} W^{+}$ & Not available & Not available \\
\hline$\tilde{b}_{1} \rightarrow b \tilde{\chi}_{1}^{0}$ & {$[87]$} & {$[88]$} \\
$\tilde{b}_{1} \rightarrow \tilde{t}_{1} W^{-}$ & Not available & Not available \\
$\tilde{t}_{1} \rightarrow c \tilde{\chi}_{1}^{0}$ & {$[89]$} & {$[90]$} \\
\hline$\tilde{t}_{1} \rightarrow b f \bar{f}^{\prime} \tilde{\chi}_{1}^{0}$ & {$[89,91]$} & Not available \\
\hline
\end{tabular}

Table 1. List of references for various experimental analyses at the LHC relevant for the present work. See text for the constraints extracted from these references.

which is kinematically disfavored in our scenario. Thus, the bounds obtained there are not applicable to our present study. Constraint on $\tilde{b}_{1}-\tilde{\chi}_{1}^{0}$ mass-plane has been derived in reference $[87,88]$ assuming $\tilde{b}_{1}$ always decaying to $b \tilde{\chi}_{1}^{0}$. As noted earlier, for varied reasons, this may not be the most favored mode of decay for $\tilde{b}_{1}$ in our case and hence the constraint would be relaxed. Reference [89] presents dedicated studies on the production of a pair of $\tilde{t}_{1}$ followed by their FCNC-decays to charm quarks and the LSP using both charm-tag and monojet-like selection (a hard jet originating in the initial state radiation). The latter technique is also employed in reference [90] to constrain the $m_{\tilde{t}_{1}}-m_{\tilde{\chi}_{1}^{0}}$ plane for small masssplit between these two states. Again, in references [89, 91], such a monojet-like search is adopted to probe the parameter space with highly mass-degenerate $\tilde{t}_{1}$ and $\tilde{\chi}_{1}^{0}$ that leads to four-body decays of $\tilde{t}_{1}$ discussed earlier. Table 1 serves as a ready-reckoner for these experimental analyses.

\subsubsection{Scenario with a bino-like LSP}

In the NMSSM, the lightest neutralino (LSP) is bino-like when the soft-breaking U(1) gaugino mass parameter $\left(M_{1}\right)$ is much smaller than both $\mu_{\text {eff }}$ and the NMSSM quantity $\kappa v_{S}$. For such a neutralino the bounds derived in reference $[87,88]$ will be applicable. Thus, the smallest LSP mass that we could legitimately consider is dictated by the mass of the lighter sbottom $\left(m_{\tilde{b}_{1}}\right)$ we would use. A ballpark value of $m_{\tilde{b}_{1}} \approx 400 \mathrm{GeV}$ would require $m_{\tilde{\chi}_{1}^{0}} \gtrsim 270 \mathrm{GeV}$. Once compatible ranges for $m_{\tilde{b}_{1}}$ and $m_{\tilde{\chi}_{1}^{0}}$ are chosen, we construct the benchmark scenarios by requiring the proposed hierarchy of masses: $m_{\tilde{\chi}_{1}^{0}}<m_{\tilde{t}_{1}}<$ $m_{\tilde{b}_{1}}<m_{\tilde{t}_{2}}<m_{\tilde{\chi}_{1}^{ \pm}}$. The bounds $m_{\tilde{t}_{1}}>240 \mathrm{GeV}$ for arbitrary $m_{\tilde{\chi}_{1}^{0}}, m_{\tilde{t}_{1}}>270 \mathrm{GeV}$ for $m_{\tilde{\chi}_{1}^{0}}=200 \mathrm{GeV}$ and the one for the nearly degenerate case $m_{\tilde{t}_{1}} \approx m_{\tilde{\chi}_{1}^{0}}>260 \mathrm{GeV}$ [89] all hold in such a scenario.

\subsubsection{Scenario with a singlino-like LSP}

A singlino-like neutralino-LSP is realized in the NMSSM for small values of $\kappa v_{S}[27,29,31$, $32]$. The couplings of such an LSP state to any other excitation are generically suppressed. Thus, for such an LSP, the decay rate in the mode $\tilde{b}_{1} \rightarrow b \tilde{\chi}_{1}^{0}$ can be significantly suppressed. 
This may lead to either a long-lived/metastable $\tilde{b}_{1}$ (if this is the only decay mode which is kinematically allowed) or to a reduced branching fraction for the same (in the presence of its other available decay modes). In any case, under such a circumstance, the colliderbound on the mass of the LSP that was relevant for the bino-like case (a prompt $\tilde{b}_{1} \rightarrow b \tilde{\chi}_{1}^{0}$ decay with $100 \%$ branching fraction) is not applicable. In sharp contrast to the binodominated LSP case, in our present scenario, $\tilde{b}_{1}$ would dominantly decay via $\tilde{t}_{1} W / W^{*}$. Unfortunately, as mentioned earlier, no experimental analysis seems to exist that exploits this particular decay mode to put bounds on the sbottom mass. ${ }^{7}$ Under the circumstances, to be conservative, we continue to consider $m_{\tilde{\chi}_{1}^{0}}>270 \mathrm{GeV}$. For a singlino-like LSP, both flavor-violating two-body decay $\tilde{t}_{1} \rightarrow c \tilde{\chi}_{1}^{0}$ and four-body decay of $\tilde{t}_{1}$ would get further suppressed. However, as long as the total (combined) decay width $\left(\Gamma_{\text {tot }}^{\left(\tilde{t}_{1}\right)} \gtrsim 10^{-13} \mathrm{GeV}\right)$ ensures an effectively prompt decay of $\tilde{t}_{1}$, the bounds on $m_{\tilde{t}_{1}}$ based on such a decay [89], as described above in the case of a bino-dominated LSP, apply. We would briefly look into this kind of a critical situation and its possible implications in section 4.1.

\subsection{Allowed regions of the NMSSM parameter space}

In order to have an idea how big the NMSSM parameter space consistent with the simplified scenario proposed in figure 4 and compatible with some theoretical and current experimental constraints is, we undertake a thorough scan of the same using NMSSMTools. The ranges for various parameters scanned over are taken from equation (2.9). The Fortran code NMHDECAY $[58,59,94]$ in NMSSMTools computes the masses, couplings and decay widths of all Higgs bosons of the NMSSM, and the masses of all sparticles. Again, the range of Higgs mass allowed for the scan is $m_{h}=125.1 \pm 3.0 \mathrm{GeV}$, as is default to NMSSMTools. As discussed in the previous subsection, we ensure the mass of the LSP neutralino to be around its lowest acceptable value compatible with the overall scenario we adhere to, i.e., $\sim 300 \mathrm{GeV}$. For the bino-dominated LSP, we thus fix $M_{1}=300 \mathrm{GeV}$. In the case of singlino-dominated LSP, such a mass for the LSP is obtained for relatively smaller values of $\kappa$ as the latter varies. Thus, for this case, we fix $M_{1}$ at $700 \mathrm{GeV}$. Further, to have a lighter chargino heavier than the two stop states and the lighter sbottom, we take $M_{2}$ large enough $(\sim 1.2 \mathrm{TeV})$. On the same ground, only a large enough value of $\mu,{ }^{8}$ would be compatible with our proposed scenario. Also, $M_{3}$, the masses of the sleptons and those of the squarks of the first two generations are held fixed at $3 \mathrm{TeV}$ which help evade the LHC bounds on the masses of the gluino and the sfermions straight away. As far as benifitting quantitatively in terms of the degree of finetuning, we find that, within the framework of NMSSMTools [60,61], the finetuning parameter $(\Delta)$ is in the ballpark of $\simeq 10$ (30) for $m_{\tilde{t}_{1}}=300(500) \mathrm{GeV}$ and $m_{\tilde{t}_{2}} \approx \mu=450(650) \mathrm{GeV}$, which are compatible with the simplified scenario we consider in this work. The values od $\Delta$ could be compared with the typical MSSM values which are at least a few hundred. If one is ready to give up on the simplified scenario, a smaller $\mu$ is acceptable thus improving further on the degree of finetuning.

\footnotetext{
${ }^{7}$ Phenomenology of such a decay mode at the LHC has been discussed in references [92, 93].

${ }^{8}$ Note that by such a choice we adopt a scenario where $M_{1} \ll \mu, M_{2}$ thus making way for either a bino-dominated or a singlino-dominated neutralino LSP depending on the value of the parameter $\kappa$.
} 
To adhere to the simplified scenario proposed in figure 4 , we require that $\tilde{t}_{2}$ does not have any appreciable branching fraction to $t \tilde{\chi}_{1}^{0}$. Thus, during the present scan, we ensure $m_{\tilde{t}_{2}}-\left(m_{t}+m_{\tilde{\chi}_{1}^{0}}\right)<50 \mathrm{GeV}$. Furthermore, as mentioned in section 3.1, we ensure that $m_{\tilde{t}_{2}}-m_{\tilde{t}_{1}}>m_{Z}$ so that $\tilde{t}_{2}$ could decay to $\tilde{t}_{1} Z$. Also, we restricted $m_{\tilde{t}_{1}}-m_{\tilde{\chi}_{1}^{0}}$ to $<80 \mathrm{GeV}$ so that the only decays of $\tilde{t}_{1}$ are to flavor-violating two-body mode $c \tilde{\chi}_{1}^{0}$ and/or four-body $b f \bar{f}^{\prime} \tilde{\chi}_{1}^{0}$ mode. The scan is subjected to various phenomenological constraints that are in-built in NMSSMTools. To name a few (see the webpage mentioned in reference [58]), these are the constraints from $B$-physics, radiative Upsilon decays, ALEPH constraints on $H \rightarrow A A \rightarrow 4 \tau$, Tevatron and LHC constraints on the charged Higgs sector, LHC constraints on $H / A \rightarrow \tau^{+} \tau^{-}, Z \gamma, H \rightarrow b b, Z Z, W W, \gamma \gamma, H \rightarrow A A \rightarrow 4 \mu$ and the LHC bound on the Higgs mass.

At this point a little digression to figure 1 would help understand where exactly our scenario lives in an otherwise allowed region of the NMSSM parameter space. The combined band in yellow (bino-like LSP neutralino) and purple (singlino-like LSP neutralino) is the relevant region. The lower (upper) diagonal edge arises by demanding $m_{\tilde{t}_{2}} \geq m_{\tilde{t}_{1}}+m_{Z}$ $\left(m_{\tilde{t}_{2}}-\left(m_{t}+m_{\tilde{\chi}_{1}^{0}}\right) \leq 50 \mathrm{GeV}\right)$. For the bino-like LSP case, the LSP mass is more or less fixed at $\approx 290 \mathrm{GeV}$, being determined by the choice $M_{1}=300 \mathrm{GeV}$. Consequently, $m_{\tilde{t}_{2}}$ is bound from above and this is reflected in the flat edge at the top of the yellow band. On the other hand, for the singlino-like case, the LSP mass is determined by $\kappa v_{S}$ and hence it varies in our scan. This pushes up the values of $m_{\tilde{t}_{1}}$ and $m_{\tilde{t}_{2}}$ that are consistent in our scenario. The flat edge at the top of the purple band relates to the maximum value of $m_{\tilde{t}_{2}}$ $(\leq 700 \mathrm{GeV})$ that we allow. Clearly, this is an artificial cut-off but a meaningful one that restricts us only to a relatively light pair of stops.

A SUSY explanation of the tantalizing excess (at $\sim 3.5 \sigma$ level) in the measured value of muon $(g-2)[95,96]$ over its SM prediction calls for a small smuon mass and a somewhat large $\tan \beta$, in particular, if the charginos are heavy. On the other hand, in our scenario with large values of $\lambda$ (and light stops), only small $\tan \beta(1 \lesssim \tan \beta \lesssim 5)$ complies with the allowed range of Higgs mass. Hence it is difficult to satisfy the muon $g-2$ constraint $[97,98]$ from within NMSSMTools (which allows for only a $2 \sigma$ window about the measured central value) without requiring the smuon to be so light that it becomes the LSP. However, allowing for a $\sim 2.5 \sigma$ downward fluctuation (i.e., only a smaller excess over the SM) could easily accommodate a suitably light smuon. Note that a light smuon does not enter the phenomenology we discuss in this work unless a chargino or a heavier neutralino is lighter than, at least, the heavier stop. Furthermore, such a scenario but with a heavier spectrum would allow for a larger $\tan \beta$. In that case a heavier smuon could fit in. For simplicity, we fix the smuon mass to a high value in this work.

In the DM sector, two different experimental constraints are in reference: (i) the Planck result that restricts the range of allowed relic abundance $\left(\Omega_{c} h^{2} \simeq 0.119 \pm 0.002\right)$ [99] and (ii) the constraint on the maximum allowed spin-independent DM-nucleon elastic scattering cross section (as a function of the mass of the DM candidate) as reported by the direct detection experiment LUX [100]. These constraints can be evaded by going beyond the 
standard cosmological model and/or assuming non-thermal DM. ${ }^{9}$ Nonetheless, a somewhat relaxed version of the constraint on the relic abundance may be to respect only the upper bound on the same thus ensuring that the universe is not over-closed. We also observe that the LUX bound is easily satisfied almost over the entire region of the parameter space of our interest, for both bino- and singlino-dominated LSP cases. Rather heavy $(\sim 3 \mathrm{TeV})$ squarks from the lighter generations help keep the spin-independent DM-nucleon elastic scattering cross section low enough for the purpose. As for the constraint related to the non-appearance of Landau pole (up to the grand unification scale $\sim \mathcal{O}\left(10^{16}\right) \mathrm{GeV}$ ), this again can be overlooked in the scheme of the so-called $\lambda$ SUSY $[39,55,104,105]$ where large values of $\lambda$ (up to 2) at the weak scale can be allowed.

In the light of the above discussion, we adopt the following strategy to understand the impacts of incorporating the constraints pertaining to relic-abundance and Landau pole only. In figure 5 and 6 we present the results of some kind of a "constraint-flow" analysis for the cases with bino- and singlino-dominated LSP, respectively. These demonstrate the outcomes of imposing these constraints successively thus shedding light on their respective impacts. In the case of the allowed range for relic abundance we use the one incorporated in the NMSSMTools, i.e., $0.107 \leq \Omega_{c} h^{2} \leq 0.131$. The plots in these two figures are drawn in the $\lambda-m_{\tilde{t}_{2}}$ plane with the values of the parameter $\kappa$ represented by the color-code defined in the adjacent color-palettes. In both figures, the plots on the top, left corners delineate the regions allowed after imposing all the constraints (including the experimentally allowed range of the mass of the Higgs boson) discussed earlier except for the ones related to the DM relic abundance and the Landau pole. This plot in each figure serves as the 'reference' with respect to which the effects of the latter two constraints are studied.

For the bino-dominated LSP (figure 5) we require the bino-admixture in the LSP to be $\geq 95 \%$. The reference plot clearly reveals that rather light stops are only compatible with large values of $\lambda$. This is just an artifact of the basic paradigm we are exploiting in this work, i.e., larger $\lambda$ compensates for lower stop masses to have the mass of the SM-like Higgs boson within the experimentally allowed range. Meanwhile, $\kappa$ can attain values ranging over moderately low to the highest value allowed in our scan. The sharp, flat edge at the top of the region indicates the largest mass for $\tilde{t}_{2}$ which is consistent with the scenario we are working in (i.e., $m_{\tilde{t}_{2}}-m_{\tilde{\chi}_{1}^{0}} \leq m_{t}+50 \mathrm{GeV}$ ).

Similarly, for a singlino-dominated LSP (figure 6) we demand the singlino-component in the LSP should be $\geq 95 \%$. The reference plot has more or less a uniform density for $\lambda \gtrsim 0.65$ and $400 \mathrm{GeV} \lesssim m_{\tilde{t}_{2}} \lesssim 700 \mathrm{GeV}$. Low values of $\kappa$ (up to $\kappa \simeq 0.3$ ) are only consistent since these naturally render the LSP singlino-dominated as long as $\kappa v_{S}=\kappa \mu_{\text {eff }} / \lambda \ll M_{1,2}$. Note that as $\kappa v_{S}$ increases within this range, the mass of the singlino dominated LSP also increases. This is in contrast with the bino-dominated LSP case described earlier where the

\footnotetext{
${ }^{9}$ For example, in the presence of non-thermal production of the DM candidate, the constraint on the DM annihilation cross-section obtained by assuming thermal production of DM can be evaded [101-103]. Further, the presence of a lighter gravitino or axino would invalidate the constraints on the lightest neutralino as the DM candidate. In the presence of other DM components (from a hidden sector, for example), the same constraints would again be relaxed. All these possibilities would make little or even no difference to the collider signatures we discuss in this work (and may not even have their own distinctive signatures).
} 

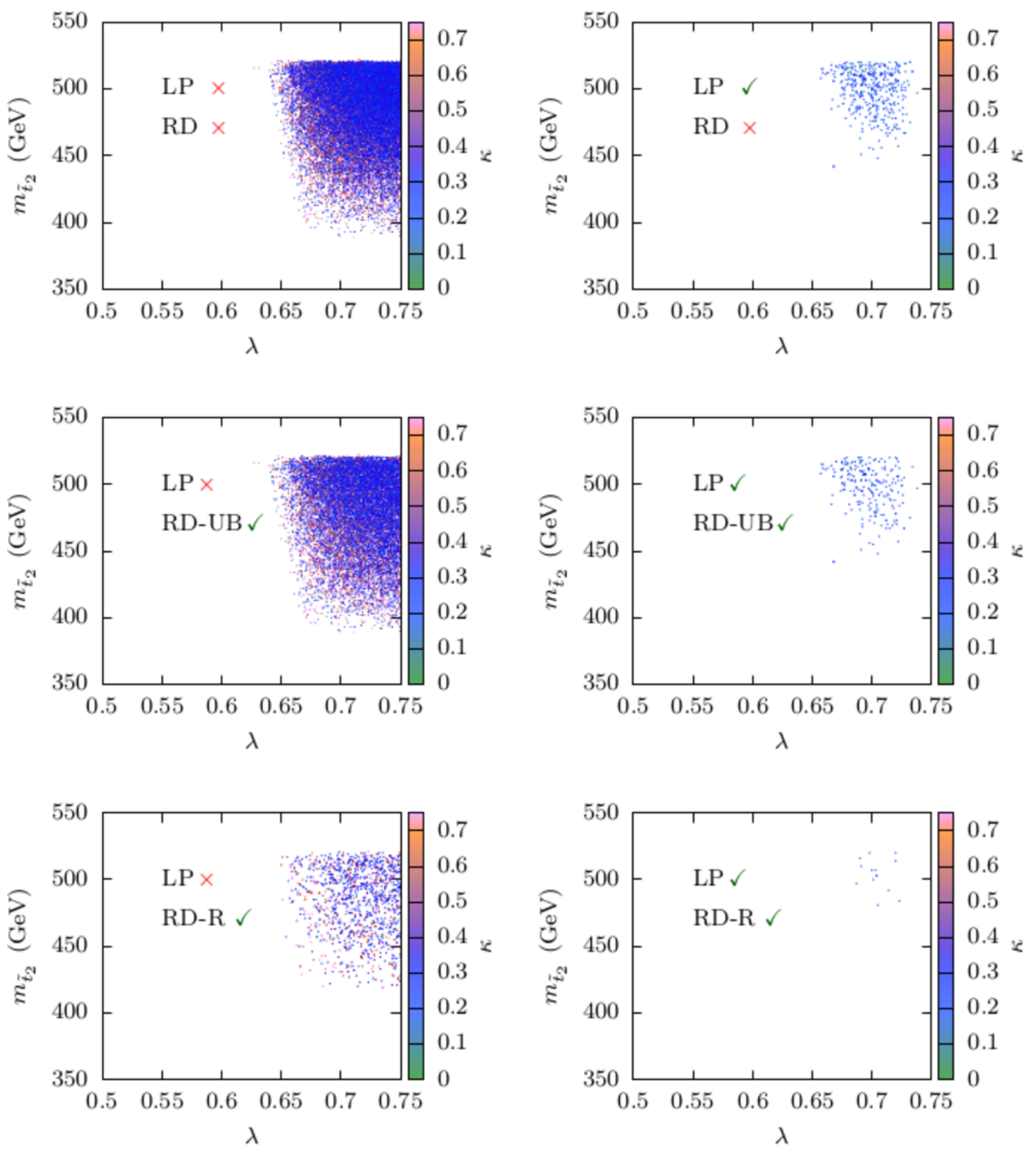

Figure 5. Scatter plots depicting the regions in the $\lambda-m_{\tilde{t}_{2}}$ plane compatible with the simplified scenario we consider and for the case of a bino-dominated LSP. All major electroweak constraints (including those for the Higgs-sector observables) as incorporated in the package NMSSMTools are considered except for the one related to muon $(g-2)$. The values of $\lambda$ and $\kappa$ are at the fixed scale of the common soft mass of the light-flavored squarks. The legends 'RD', 'RD-UB' and 'RD-R' stand for 'relic density', 'relic density upper bound' and 'relic density range', respectively. The legend 'LP' stands for 'Landau pole' related requirements. The symbols ' $\checkmark$ ' and ' $x$ ' against these legends convey whether these particular types of constraints are imposed or not. See text for details.

LSP mass gets more or less fixed by fixing the MSSM parameter $M_{1}$. An increasing mass of the LSP drives (slides) the entire spectrum of our simplified scenario to the heavier side.

We now move on to impose the constraint from the DM relic abundance as reported by the Planck experiment. In addition, we would also demand absence of Landau poles 

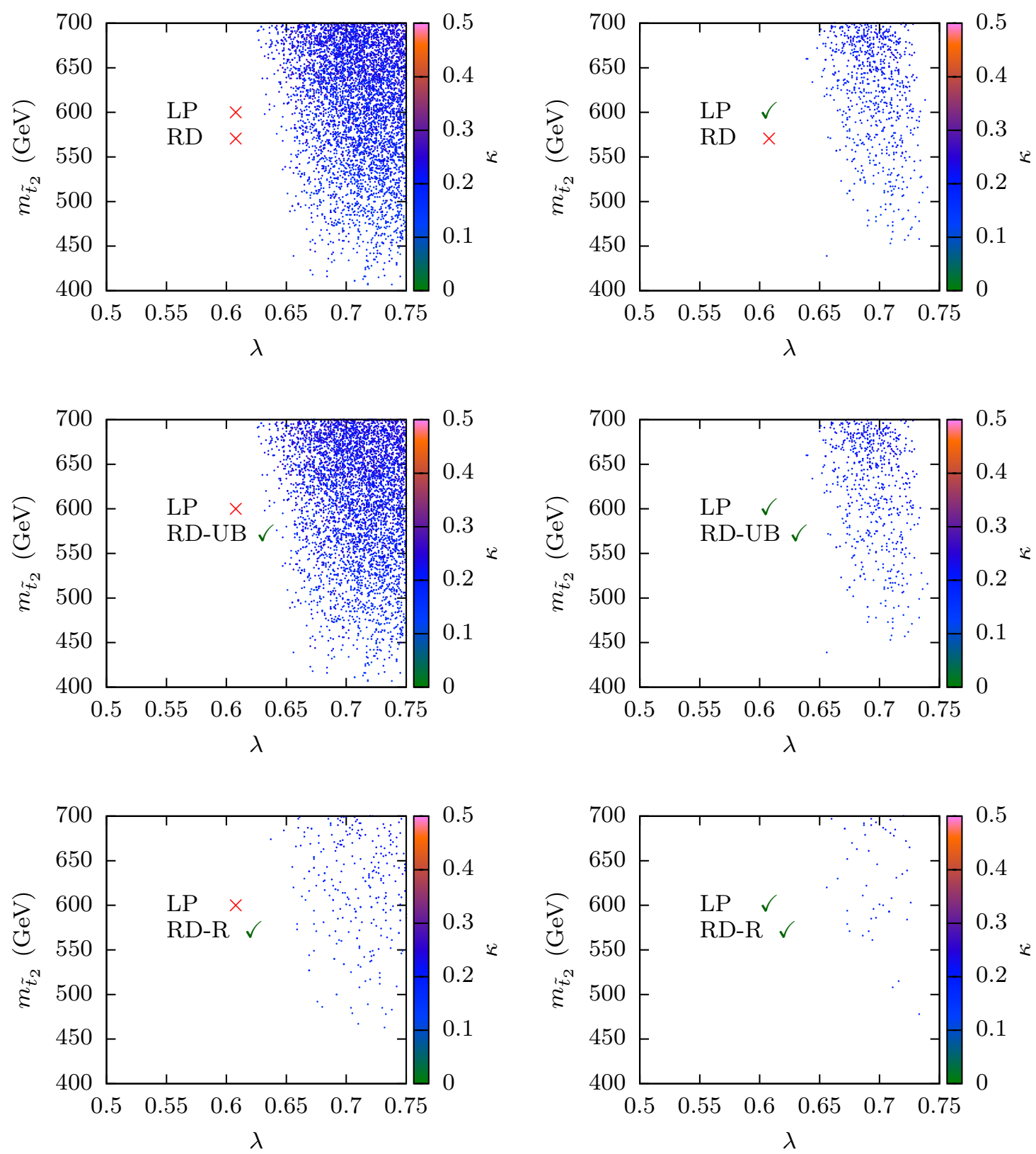

Figure 6. Same as in figure 5 but for the case of a singlino-dominated LSP.

in the evolution of the parameters like $\lambda, \kappa$ and the top and the bottom quark Yukawa couplings up to the unification scale which is around $10^{16} \mathrm{GeV}$. Along the rows we show the allowed regions on further impositions of the relic density upper bound of $\Omega_{c} h^{2}=0.131$ (the loose criterion; second row) and the constrained range for the same as implemented in NMSSMTools and as mentioned earlier (the tight criterion; last row). On the other hand, for the plots in the first column, the Landau pole-related constraint is not imposed while for the ones in the second column this is incorporated.

It can be clearly seen that as we go from the top to the bottom of these figures, the 'loose' demand on the relic abundance does not affect the otherwise allowed regions of 
the NMSSM parameter plane in any significant way. However, incorporating the 'tight' criterion on the relic abundance results in shrinking of the allowed parameter plane towards larger values of $\lambda$ and $m_{\tilde{t}_{2}}$ in the case of a bino-dominated LSP. That the smaller values of $m_{\tilde{t}_{2}}$ get less preferred is understandable as follows. Allowing for a range of relic abundance, which is now also bounded from below, implies that we cut off the low-abundance region, i.e., the region with higher overall annihilation rate. This is achieved when the stop masses are increased since these reduce the LSP- $\tilde{t}_{1}$ coannihilation rate in the one hand and the $\tilde{t}_{1}$ and $\tilde{t}_{2}$ mediated LSP annihilation on the other.

As for the singlino-dominated LSP, a careful study reveals that the 'tight' criterion does not alter the already-allowed region too much. The only effect it has is in the form of lowering the density of points in the allowed region (which is a natural effect when a new constraint is imposed). This may be understood in terms of the fact that unlike in the bino-like LSP case, dominant annihilation and coannihilation modes of the singlinoLSP do not involve $\tilde{t}_{2}$. Hence, as the 'tight' criterion does, putting a lower bound on the relic abundance does not have much effect on the already allowed region of the NMSSM parameter plane.

Next we discuss the impact of imposing the demand related to Landau pole (as we move from the first column to the second). In both bino- and singlino-dominated LSP cases, this turns out to be more restrictive compared to the ones pertaining to the relic abundance. Irrespective of the nature of the LSP, this constraint puts an upper bound on $\lambda .{ }^{10}$ For a bino-dominated LSP, the Landau pole constraint pushes up the stop masses by an appreciable extent.

In figure 7 we present the projected regions in different parameter planes which are relevant for the current study. We opt for a reasonably conservative scenario where the Landau pole related constraint is enforced in the strictest sense but only the upper bound, rather than the allowed range, of the relic abundance is required to be satisfied. Plots in the left column present the cases with bino-dominated LSP while the ones in the right column illustrate the cases where the LSP is singlino-dominated. The top row delineates the compatible region in the $m_{\tilde{t}_{1}}-m_{\tilde{t}_{2}}$ in our simplified scenario, with variation of $\kappa$ being indicated by the color-code defined in the adjacent palettes. The plot on the top, left corner have the same set of points as the plot in the second row and second of column of figure 5 but now projected on a different plane. The points here populate a roughly rectangular box whose edges are determined by the hierarchy we have adopted for our scenario, the mutual mass-splits that we have allowed and the minimum LSP mass that we have chosen. On the other hand, the right plot in top row presenting the case with singlino-dominated LSP, clearly displays a correlation between $m_{\tilde{t}_{1}}$ and $m_{\tilde{t}_{2}}$ as they grow. It has been already discussed that allowing for a variation of $\kappa$ implies a change in mass of the LSP-singlino which 'slides' the entire spectrum to high mass-values. The almost uniform width of the

\footnotetext{
${ }^{10}$ The maximum value of $\lambda$ that we allow in our scan $(\lambda=0.75$, at the SUSY breaking scale given by the average mass of the squarks from the first two generations, i.e., $3 \mathrm{TeV}$ ) is, in the first place, motivated by the Landau-pole constraint. As discussed earlier in this section, a scenario like $\lambda$ SUSY considers large values of $\lambda$ (up to 2) at the weak scale without jeopardizing the unification of gauge couplings at a high scale [104].
} 
band along the diagonal results from a very restrictive mutual splittings that are enforced among the low-lying states to comply with our simplified scenario. One may like to refer back to the yellow (bino-dominated LSP) and purple (singlino-dominated LSP) bands in figure 1 essentially from which the left and the right plots in the first row of figure 7 are derived by imposing further constraints. Also, note that in both the cases $\kappa$ remains small. In the bino-dominated (left) case, $\kappa$ could have been larger were it not for the fact that it is prohibited by demanding absence of Landau pole.

From the plots in the bottom row we clearly see a strong correlation between the mutual mass-splits: an increasing split between $m_{\tilde{b}_{1}}$ and $m_{\tilde{t}_{1}}$ results in a shrinking of the split between $m_{\tilde{t}_{2}}$ and $m_{\tilde{b}_{1}}$. This is natural because of the imposed hierarchy $m_{\tilde{t}_{1}}<m_{\tilde{b}_{1}}<m_{\tilde{t}_{2}}$ with a restricted maximum splitting between $m_{\tilde{t}_{2}}$ and LSP mass. Since the LSP mass is essentially determined by the fixed value of $M_{1}(=300 \mathrm{GeV})$ in the bino-dominated case, the left figure displays a uniform (blue) value that $m_{\tilde{t}_{2}}$ could take as all other parameters vary in the scan. On the other hand, for the reason discussed above, the case with a singlinodominated LSP allows for a range of $m_{\tilde{t}_{2}}$ (blue and reddish points) as $\kappa$ varies. One can notice that somewhat lower mutual splittings are allowed simultaneously in this case.

\subsection{Choosing the benchmark scenarios}

In table 2, we present three benchmark scenarios that satisfy the simplified situation demonstrated in figure 4: two of them are for the bino-like LSP case and one is for the singlino-like LSP case. All three scenarios are confronted by the constraints coming from various different search modes at the LHC experiments via the package CheckMATE (v1.2.0) [106] (to the extent the corresponding analyses are available with the said package) and are found to pass them. These points also satisfy the upper bound on relic density and safe against Landau pole. All three benchmark points satisfy the LUX constraint. However, as discussed earlier, the constraint from muon $(\mathrm{g}-2)$ is not incorporated.

The two (pure) bino-like LSP scenarios, BP1 and BP2 (with 99\% bino-admixture), differ only in the values of the soft mass parameters for the U(1) gaugino and those for the doublet and the up-type singlet squarks from the third generation (i.e., $m_{\tilde{Q}_{3}}$ and $m_{\tilde{U}_{3}}$ ). Slightly different values for the trilinear soft SUSY breaking term $A_{t}$ have been chosen. The purpose of presenting two such benchmark points in the bino-dominated LSP scenario is just to demonstrate that significantly different branching fractions in the cascades are attainable which would have drastic bearings for the final states. Such variations could also be achieved by varying some of the NMSSM inputs simultaneously and hence the specific choices of the input parameters need not be over-stressed. Nonetheless, some aspects of these choices, like largeness of $\lambda$ and an appropriate intermediate value for $\kappa$ (thus, together, ensuring constraints pertaining to non-appearance of Landau pole up to the unification scale), not so large values of the soft masses $m_{\tilde{Q}_{3}}$ and $m_{\tilde{U}_{3}}$ are very much of a defining nature for the scenario under consideration. It is observed that not too different values of $m_{\tilde{Q}_{3}}$ and $m_{\tilde{U}_{3}}$ can be afforded since the scenario we are considering is intrinsically of a 'compressed' nature. Naturally, the relevant spectra involving the stop, sbottom and the LSP are not so different. Indeed, it is interesting to note that the decay branching fractions of the stop and the sbottom squarks to the available modes are markedly different for BP1 


\begin{tabular}{|c|c|c|c|}
\hline \multirow{2}{*}{$\begin{array}{c}\text { Input } \\
\text { Parameters }\end{array}$} & \multicolumn{2}{|c|}{ Bino-like LSP } & Singlino-like LSP \\
\hline & $\mathrm{BP} 1$ & $\mathrm{BP} 2$ & BP3 \\
\hline$\lambda$ & 0.70 & 0.70 & 0.69 \\
\hline$\kappa$ & 0.20 & 0.20 & 0.11 \\
\hline$A_{\lambda}(\mathrm{GeV})$ & 1800 & 1730 & 1950 \\
\hline$A_{\kappa}(\mathrm{GeV})$ & -135 & -135 & -135 \\
\hline$\mu_{\text {eff }}(\mathrm{GeV})$ & 900 & 900 & 900 \\
\hline $\tan \beta$ & 2 & 2 & 2 \\
\hline$A_{t}(\mathrm{GeV})$ & -100 & -250 & -200 \\
\hline$M_{1}(\mathrm{GeV})$ & 294 & 294 & 600 \\
\hline$m_{\tilde{Q}_{3}}(\mathrm{GeV})$ & 794 & 805 & 800 \\
\hline$m_{\tilde{U}_{3}}(\mathrm{GeV})$ & 834 & 836 & 832 \\
\hline$m_{\tilde{D}_{3}}(\mathrm{GeV})$ & 3000 & 3000 & 3000 \\
\hline Observables & $\mathrm{BP} 1$ & $\mathrm{BP} 2$ & $\mathrm{BP} 3$ \\
\hline $\begin{array}{l}\text { Bino/Singlino } \\
\text { fraction in the LSP }\end{array}$ & 0.99 & 0.99 & 0.99 \\
\hline$m_{h}(\mathrm{GeV})$ & 123.3 & 125.0 & 123.0 \\
\hline$m_{\tilde{t}_{2}}(\mathrm{GeV})$ & 480.8 & 518.6 & 500.4 \\
\hline$m_{\tilde{b}_{1}}(\mathrm{GeV})$ & 372.8 & 404.0 & 387.9 \\
\hline$m_{\tilde{t}_{1}}(\mathrm{GeV})$ & 320.5 & 312.1 & 304.2 \\
\hline$m_{L S P}(\mathrm{GeV})$ & 290.4 & 290.4 & 278.8 \\
\hline $\operatorname{BR}\left(\tilde{t}_{2} \rightarrow \tilde{t}_{1} Z\right)$ & 0.57 & 0.67 & 0.67 \\
\hline $\operatorname{BR}\left(\tilde{t}_{2} \rightarrow \tilde{b}_{1} W^{+}\right)$ & 0.39 & 0.30 & 0.32 \\
\hline $\operatorname{BR}\left(\tilde{b}_{1} \rightarrow \tilde{t}_{1} W^{-} / W^{-*}\right)$ & 0.05 & 0.90 & $\sim 1$ \\
\hline $\mathrm{BR}\left(\tilde{b}_{1} \rightarrow b\right.$ LSP $)$ & 0.95 & 0.10 & $\sim 0$ \\
\hline$\Gamma_{\tilde{t}_{1}}(\mathrm{GeV})$ & $3.4 \times 10^{-12}$ & $5.2 \times 10^{-13}$ & $4.8 \times 10^{-14}$ \\
\hline $\mathrm{BR}\left[\tilde{t}_{1} \rightarrow c \tilde{\chi}_{1}^{0}\right]$ & 0.15 & 0.52 & 0.99 \\
\hline $\mathrm{BR}\left[\tilde{t}_{1} \rightarrow b f \bar{f}^{\prime} \tilde{\chi}_{1}^{0}\right]$ & 0.85 & 0.48 & 0.01 \\
\hline$\sigma\left(p p \rightarrow \tilde{t}_{2} \tilde{t}_{2}^{*}\right)_{\mathrm{LHC} 13}(\mathrm{fb})$ & 633 & 412 & 503 \\
\hline$\sigma\left(p p \rightarrow \tilde{t}_{2} \tilde{t}_{2}^{*}\right)_{\mathrm{LHC} 14}(\mathrm{fb})$ & 804 & 523 & 641 \\
\hline
\end{tabular}

Table 2. Benchmark set of input parameters for the cases with bino-dominated (BP1 and BP2) and the singlino-dominated (BP3) LSP along with the resulting spectra and the branching fractions for the important decay modes including those for the lighter stop (see figure 10). The total decay width of the lighter stop (see figure 9) and the production cross sections of the $\tilde{t}_{2}$-pair (at the NLO+NLL accuracy, for $13 \mathrm{TeV}$ (LHC-13) and $14 \mathrm{TeV}$ (LHC-14) LHC runs; see figure 11) are also indicated. Soft masses of the squarks from the first two generations, those for all the sleptons and the $\mathrm{SU}(3)$ gaugino mass, $M_{3}$ are set to $3 \mathrm{TeV}$. The $\mathrm{SU}(2)$ gaugino mass $M_{2}$ is set to $1.2 \mathrm{TeV}$. The SM-like Higgs boson happens to be the lightest Higgs boson for all the three benchmark points. See text for details. 

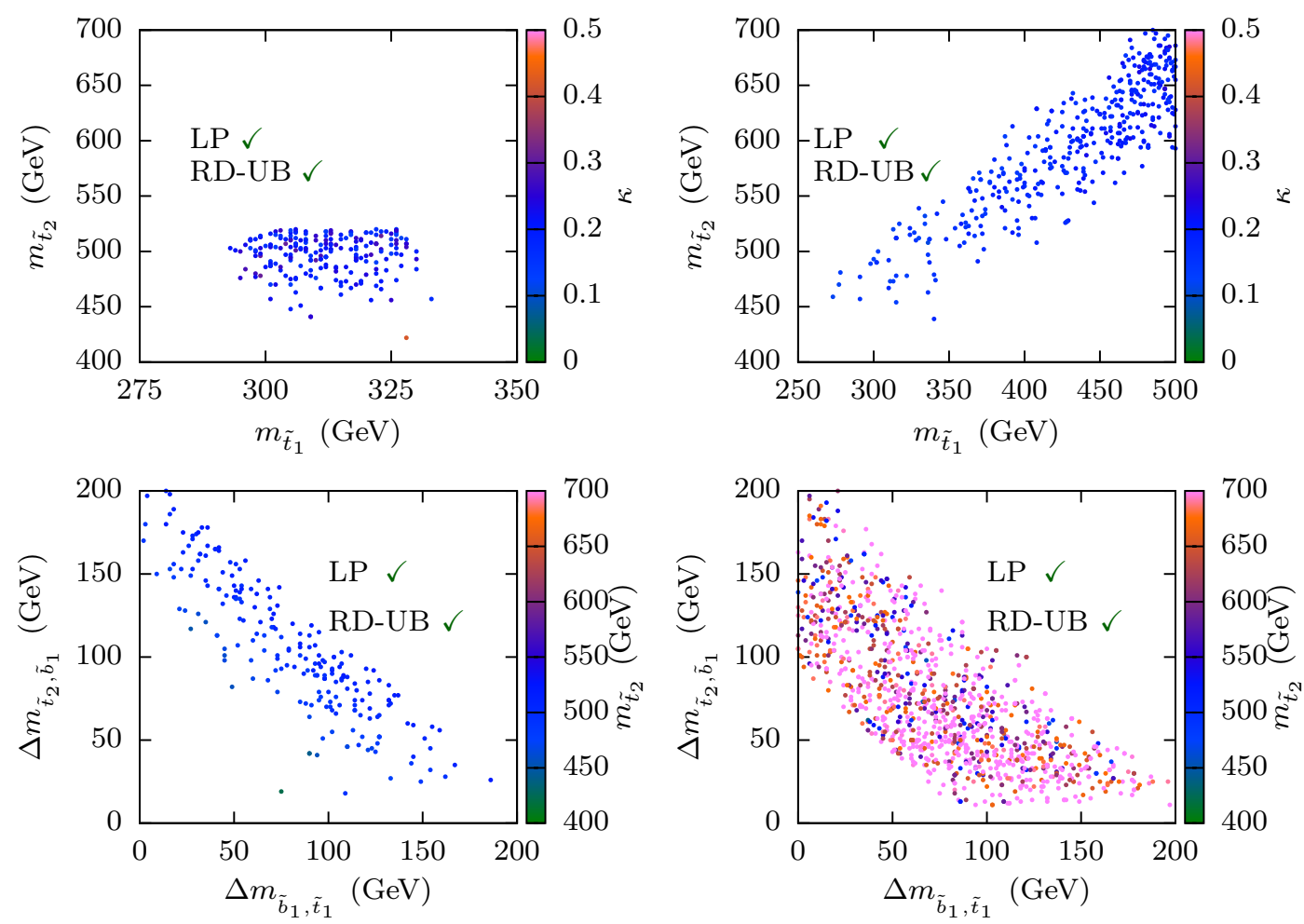

Figure 7. Scatter plots depicting the regions in the $m_{\tilde{t}_{1}}-m_{\tilde{t}_{2}}$ plane (upper panel) and in the plane of two mutual splittings: $\Delta m_{\tilde{b}_{1}, \tilde{t}_{1}}$ and $\Delta m_{\tilde{t}_{2}, \tilde{b}_{1}}$ (lower panel). As before, the left column represents the case of bino-dominated LSP while the right column does the same for singlino-dominated LSP case. In each plot and for each scatter point, the value of $\kappa$ (upper panel) or $m_{\tilde{t}_{2}}$ (lower panel) is indicated by the color code defined in the adjacent color-palette. For all the cases, the scans are subjected to the constraint pertaining to the absence of Landau pole and that on the maximum of DM relic density.

and BP2. As can be expected (and to be exploited later in this work), these differences would inevitably show up in the strengths of different possible final states through which such scenarios can be probed. The bottom-line is that the search channels can be rather sensitive to the actual spectrum in such compressed scenarios and this can be expected to be a somewhat generic feature.

BP3 presents a (an almost pure) singlino-like LSP scenario. Singlino-domination is ensured by a relatively low value of $\kappa$ and with a large value of $M_{1}$. In this case, the second lightest neutralino state becomes bino-dominated as long as $\mu_{\text {eff }}$ and $M_{2}$ are much larger than $M_{1}$, which is the case for BP3. Note that the choice $M_{1}=600 \mathrm{GeV}$ makes $m_{\chi_{2}^{0}}$ minimally large $\left(\sim M_{1}=600 \mathrm{GeV}\right)$ that ensures the setup we like to adhere to in the sense that a new decay mode like $\tilde{t}_{2} \rightarrow t \chi_{2}^{0}$ does not open up. Hence such a choice would not broadly affect the collider phenomenology we address in this work while a richer phenomenology could be envisaged with a lighter bino-like neutralino on its own right.

Note that the spectrum for BP3 is not again very different from the bino-dominated cases $(\mathrm{BP} 1$ and $\mathrm{BP} 2)$. In all the three cases, $m_{\tilde{t}_{2}} \sim 500 \mathrm{GeV}, m_{\tilde{b}_{1}} \sim 400 \mathrm{GeV}, m_{\tilde{t}_{1}} \gtrsim$ 
$300 \mathrm{GeV}$ and $m_{L S P} \sim 270-300 \mathrm{GeV}$. The essential difference between the scenario of BP3 and those of $\mathrm{BP} 1$ and $\mathrm{BP} 2$ is that in BP3 $\tilde{b}_{1}$ can only (for all practical purposes) decay to states involving an on- or off-shell $W$-boson. This is since for BP3, singlino-domination in the LSP efficiently suppresses the coupling $\tilde{b}_{1}-b-\tilde{\chi}_{1}^{0}$ to an insignificant level. In fact, in the singlino limit, such a suppression is generic and this affects the decay of the NLSP (next to LSP) stop $\left(\tilde{t}_{1}\right)$ as well. However, the singlino-like LSP state being the only state which $\tilde{t}_{1}$ could decay to, the pertinent issue is to what extent its decay width is affected by the suppressed coupling. As we will discuss later, this could clearly have implications for the LHC. The important branching fractions for the involved states are presented at the end of table 2 for the three benchmark points. The extent of contrast in these branching fractions is apparent. These are instrumental in shaping up the characteristic final states for each of these scenarios through which they can be probed at the LHC.

Further, it is observed that only low values of $\tan \beta$ are consistent with the scenario we consider that requires larger $\lambda$. This corroborates the findings of reference [20] with the exception that in our case only the lightest Higgs state can be the SM-like one. This fact is intimately related to our choice of the LSP mass $(\sim 300 \mathrm{GeV})$; be it a bino-like or a singlino-like LSP, and that of $\mu_{\text {eff }}$. This can be roughly understood as follows. As discussed in the beginning of section 2.2, the squared mass of the SM-like (CP-even) Higgs state is given by 2.5 while the same for the singlet-like CP-even Higgs boson is of the form $m_{s s}^{2}=\kappa v_{S}\left(A_{\kappa}+4 \kappa v_{S}\right)[6,38]$. Note that the entry for the singlino mass as shown in the neutralino mass matrix, given by equation (2.11), is $\mathcal{M}_{0}^{55}=2 \kappa v_{S}$. The product $\kappa v_{S}$ thus appears in both $m_{s s}^{2}$ and $\mathcal{M}_{0}^{55}$. A bino-like LSP with a mass $\sim 300 \mathrm{GeV}$ can now be obtained by making the mass of the singlino-like neutralino to be much larger, i.e., by requiring $2 \kappa v_{S} \gg 300 \mathrm{GeV}$. This in turn makes the mass of the singlet-like CP-even Higgs boson $\left(m_{s s}\right)$ heavier than the SM-like Higgs boson thus making the latter the lightest Higgs state. On the other hand, for a singlino-like neutralino with mass around $300 \mathrm{GeV}, \kappa v_{S}$ should have a low to moderate value and it is a priori not impossible to have the lightest Higgs boson to be singlet-like and the SM-like Higgs boson becoming the second lightest Higgs boson. However, our requirement of a somewhat large $\mu_{\mathrm{eff}}=\lambda v_{S}\left(>m_{\tilde{t}_{2}}\right)$ ensures a large $\kappa v_{S}$ (for a given $\lambda \lesssim 1$ ) thus lifting up $m_{s s}$. This renders the singlet-like Higgs boson heavier. Hence again the SM-like Higgs boson turns out to be the lightest Higgs state.

\section{A pair of light stops at the LHC}

In this section, we present the phenomenology of a pair of light stops produced directly at the LHC by studying the cascades they undergo in a 'simplified' NMSSM scenario described earlier. We adhere to the broad scenario of figure 4. All three benchmark points discussed in section 3.3 conform to this. There the choice of input parameters respects the latest experimental bounds. However, as already clarified in section 3.3, we do not subject these points to the observed value of muon $(g-2)$ and those pertaining to the DM sector.

\subsection{Cascade decay of the heavier stop and possible final states}

At the LHC, characteristic signatures of such a scenario with two light stops are triggered by the production and decay of the heavier stop $\left(\tilde{t}_{2}\right)$. The lighter stop, being the NLSP 

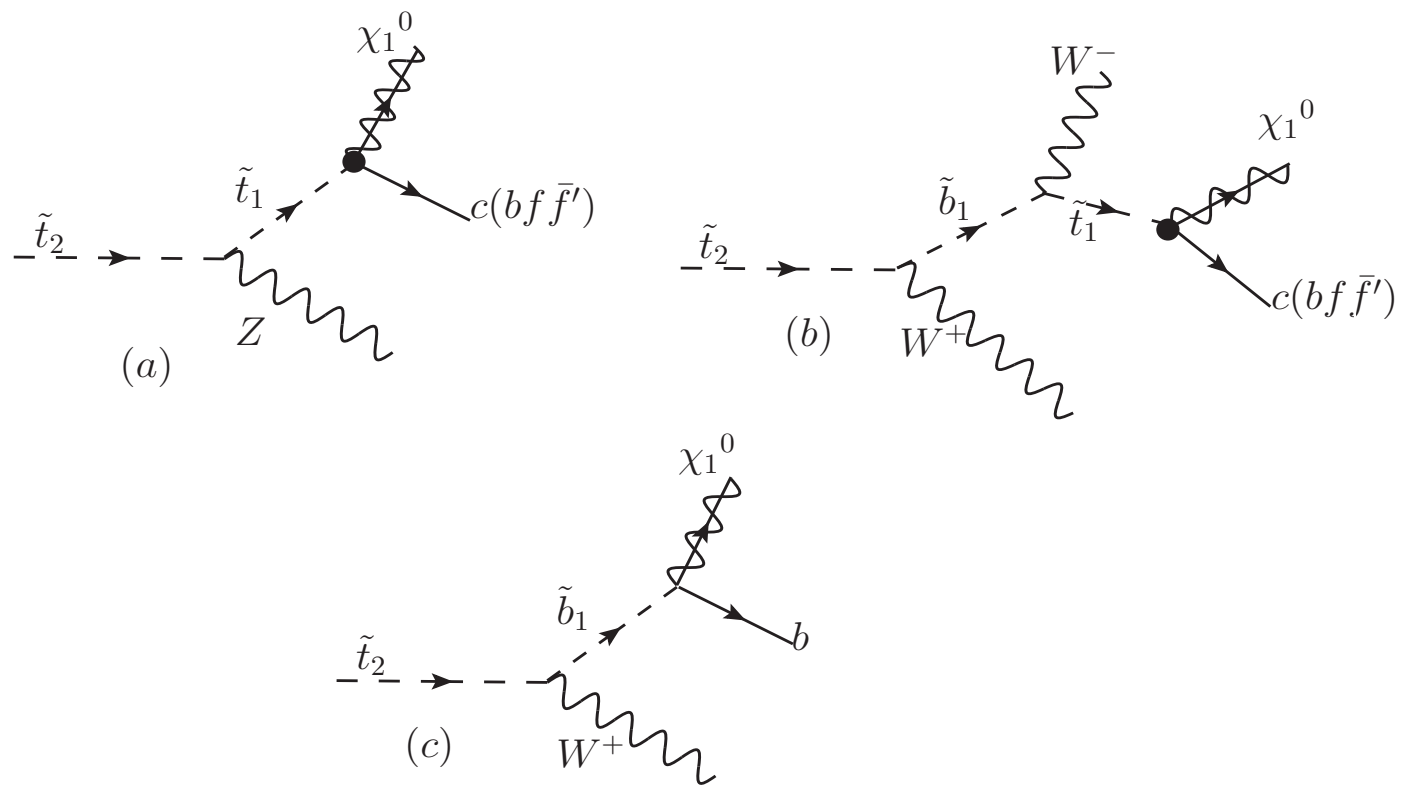

Figure 8. Possible cascades of $\tilde{t}_{2}$ in the simplified scenario we consider. Competing two-body (to $c \tilde{\chi}_{1}^{0}$ ) and four-body (to $b f \bar{f}^{\prime} \tilde{\chi}_{1}^{0}$ ) decays of $\tilde{t}_{1}$ are also considered for which the effective vertices are presented as black filled blobs.

and thus could decay only in a very restricted way, essentially plays a supporting role. The strong production cross section (which by far dominates) of the pair $\tilde{t}_{2} \tilde{t}_{2}^{*}$ is a simple function of $m_{\tilde{t}_{2}}$. Once produced, $\tilde{t}_{2}$ could cascade to the LSP in three possible ways as shown in figure 8 . Figures $8(\mathrm{a})$ and $8(\mathrm{~b})$ also indicate that $\tilde{t}_{1}$ could have FV and $4 \mathrm{~B}$ decays. These could potentially compete in our present scenario. We would briefly discuss the phenomenological implications of such possibilities later in this subsection. For convenience, we show in table 3 the final states that each of these branches leads to. We denote a pair of leptons originating in the decay of a $Z$-boson by a brace underneath. The forward slashes separate the possible alternate final states that the decays of $Z$ - or a $W$-boson(s) (appearing in a cascade) may result in. Thus, the final states presented in the third column of table 3 serve as the seeds when constructing the overall final states arising from cascades of a pair of $\tilde{t}_{2} \tilde{t}_{2}^{*}$.

In table 4 we display the different finals states constructed out of possible (6 in all) cascade-combinations of $\tilde{t}_{2}$ and $\tilde{t}_{2}^{*}$. The combinations of individual cascades $(a, b$ and $c)$ in the first column refer directly to the diagrams/cascades presented in figure $8 /$ table 3 . For clarity, we divide the final states arising from each combination of cascades presented in the first column into two parts: (i) states appearing in the decays of $\tilde{t}_{1} / \tilde{b}_{1}$ in the last phase of the SUSY cascade that include jets comprising of bottom, charm and other light quarks, leptons along with missing energy carriers like the neutrinos and the LSP, (ii) the states (jets, leptons and neutrinos) arising in the decays of $Z$ - and/or $W$-bosons appearing in the cascade decays of $\tilde{t}_{2}$. As mentioned earlier, braces under a lepton pair indicate that they are coming from the decay of a $Z$-boson. Note that although there is a multitude 


\begin{tabular}{|c|c|c|}
\hline Diagram & Cascade modes of $\tilde{t}_{2}$ & Cascade products \\
\hline \multirow{2}{*}{$(a)$} & $\tilde{t}_{2} \rightarrow \tilde{t}_{1} Z, \quad \tilde{t}_{1} \rightarrow\left(c / b f \bar{f}^{\prime}\right) \tilde{\chi}_{1}^{0}$ & $(\underbrace{\ell \bar{\ell}} / 2 j)+1 c / 1 b+\mathbb{E}_{T}$ \\
$(b)$ & $\tilde{t}_{2} \rightarrow \tilde{b}_{1} W^{+}, \quad \tilde{b}_{1} \rightarrow \tilde{t}_{1} W^{-}, \quad \tilde{t}_{1} \rightarrow\left(c / b f \bar{f}^{\prime}\right) \tilde{\chi}_{1}^{0}$ & $(\ell \bar{\ell} / 1 \ell(\bar{\ell})+2 j / 4 j)+1 c / 1 b+\mathbb{E}_{T}$ \\
$(c)$ & $\begin{array}{c}\tilde{t}_{2} \rightarrow \tilde{b}_{1} W^{+}, \quad \tilde{b}_{1} \rightarrow b \tilde{\chi}_{1}^{0} \\
(\text { only if the LSP is not singlino-like) }\end{array}$ & $(1 \ell / 2 j)+1 b+\mathbb{E}_{T}$ \\
\hline
\end{tabular}

Table 3. Possible decay chains of $\tilde{t}_{2}$ following the cascade diagrams shown in figure 8 . The third column presents the possible final states arising from the respective cascades. For the decay of $\tilde{t}_{2}^{*}$, each entry needs to be charge-conjugated.

of possible final states, these are all at the parton-level. In our actual collider simulation which we describe in the next subsection, the multi-jet final states are treated to a very good degree of sophistication by using advanced jet-related techniques. These render only final states with limited jet multiplicities to be of practical interest.

Before we end this subsection, a brief discussion over the possible decays of $\tilde{t}_{1}$ would be in place. As pointed out already, $\tilde{t}_{1}$ could undergo the two-body FV decay to $c \tilde{\chi}_{1}^{0}$ and the $4 \mathrm{~B}$ decay to $b f \bar{f}^{\prime} \tilde{\chi}_{1}^{0}$. Recently, the implications of the competition between these decay modes have been much appreciated [107-111], in particular, in the context of compressed scenarios in the MSSM with a light $\tilde{t}_{1} .{ }^{11}$ These are subsequently followed up by the LHC collaborations [89, 91].

Our concern is over a possibly longer lifetime of $\tilde{t}_{1}$ which would have crucial effect on its phenomenology and on the consequent experimental strategy to find them. In our scenario, this may happen for two different reasons. First, for an NLSP $\tilde{t}_{1}$ with a rather small masssplit with the LSP, there is a looming possibility that its decay width becomes critically suppressed by the phase space. Second, in the NMSSM, for a singlino-like LSP, the decay width of $\tilde{t}_{1}$ can be severely affected due to its suppressed (effective) couplings to the LSP. Worse could be the situation when these two issues work in tandem. In reference [115], phenomenology with non-prompt decays of (long-lived) NSLP $\tilde{t}_{1}$ has been discussed in much details where $\tilde{t}_{1}$ is either stable across the pixel or tracker detector resulting in a displaced vertex or stable over the whole detector dimension (metastable) thus leaving a charge-track. It is important to note that for a metastable $\tilde{t}_{1}$ the latest LHC analyses already exclude $m_{\tilde{t}_{1}}<900 \mathrm{GeV}$ [116] (except for a possible caveat recently pointed out in reference [117]). Hence any analysis with light $\tilde{t}_{1}$ must ensure that this does not become long-lived.

In figure 9 we show via scatter plots the individual lifetimes of $\tilde{t}_{1}$ with only FV (in red), only $4 \mathrm{~B}$ (in blue) decays and the overall lifetime (in green). These are shown for both the bino-dominated LSP (left) and the singlino-dominated LSP (right) cases. Note

\footnotetext{
${ }^{11}$ Various strategies to probe the stops squarks in difficult situations with compressed spectrum are proposed in the literature (see, for example, references [112-114] and references therein).
} 


\begin{tabular}{|c|c|c|c|}
\hline \multirow{2}{*}{$\begin{array}{c}\text { Cascade } \\
\text { combination }\end{array}$} & \multirow{2}{*}{$\begin{array}{c}\text { On-shell } \\
\text { Gauge Bosons }\end{array}$} & \multicolumn{2}{|c|}{ Final states arising from decays of a $\tilde{t}_{2} \tilde{t}_{2}^{*}$ pair } \\
\hline & & From $\tilde{t}_{1}$ decay & From $W^{ \pm} \& Z$ decays (in $\tilde{t}_{2}, \tilde{b}_{1}$ cascades) \\
\hline$(a)(a)^{*}$ & $Z Z$ & $\left.\begin{array}{rr}2 c+\tilde{\chi}_{1}^{0} \tilde{\chi}_{1}^{0} & (\mathbf{A}) \\
2 b+\ell^{+} \ell^{-} \nu \bar{\nu}+\tilde{\chi}_{1}^{0} \tilde{\chi}_{1}^{0} & (\mathbf{B}) \\
2 b+4 j+\tilde{\chi}_{1}^{0} \tilde{\chi}_{1}^{0} & (\mathbf{C}) \\
1 c+1 b+\ell \nu+\tilde{\chi}_{1}^{0} \tilde{\chi}_{1}^{0} & (\mathbf{D}) \\
1 c+1 b+j j+\tilde{\chi}_{1}^{0} \tilde{\chi}_{1}^{0} & (\mathbf{E})\end{array}\right\}$ & $2 \underbrace{\bar{\ell} \ell}, \underbrace{\overline{\ell \ell}}+2 j, \underbrace{\bar{\ell} \ell}$ \\
\hline$(b)(b)^{*}$ & $2\left(W^{+} W^{-}\right)$ & Same as above & $\begin{array}{c}2 \bar{\ell}+2 \ell, \quad 2 \bar{\ell}(\ell)+\ell(\bar{\ell})+2 j \\
\bar{\ell} \ell+4 j, \quad \mathrm{SSDL}+4 j\end{array}$ \\
\hline$(a)(b)^{*}+h . c$. & $Z\left(W^{+} W^{-}\right)$ & Same as above & $\begin{array}{c}\underbrace{\bar{\ell}}_{\bar{\ell} \ell, \overline{\ell \ell} \ell}+2 j, \quad \underbrace{\bar{\ell} \ell}+\bar{\ell}(\ell)+2 j, \\
\underbrace{\bar{\ell} \ell}+4 j\end{array}$ \\
\hline$(c)(c)^{*}$ & $W^{+} W^{-}$ & $\mathbf{B}$ and $\mathbf{C}$ & $\overline{\ell \ell}$ \\
\hline$(a)(c)^{*}+h . c$. & $Z W^{ \pm}$ & $\mathbf{B}, \mathbf{C}, \mathbf{D}$ and $\mathbf{E}$ & $\underbrace{\overline{\ell \ell}}+\bar{\ell}(\ell), \underbrace{\overline{\ell \ell}}+2 j$ \\
\hline (b) $(c)^{*}+$ h.c. & $\left(W^{+} W^{-}\right) W^{ \pm}$ & Same as above & $2 \bar{\ell}(\ell)+\ell(\bar{\ell}), \mathrm{SSDL}+2 j, \overline{\ell \ell}+2 j$ \\
\hline
\end{tabular}

Table 4. All possible final states from the decays of a pair of $\tilde{t}_{2} \tilde{t}_{2}^{*}$. Each row corresponds to specific cascades of $\tilde{t}_{2}$ and $\tilde{t}_{2}^{*}$. For each row, the set of particles appearing in the final state combines the ones from the third column (from the decays of $\tilde{t}_{1} / \tilde{t}_{1}^{*}$ ) and those from the fourth column (coming from the decays of $Z$ - and $W$-bosons produced in the cascades). Only those final states with $\geq 2$ leptons are shown. Presence of neutrinos is implied in leptonic decays of $W^{ \pm}$and when $Z$ decays invisibly.
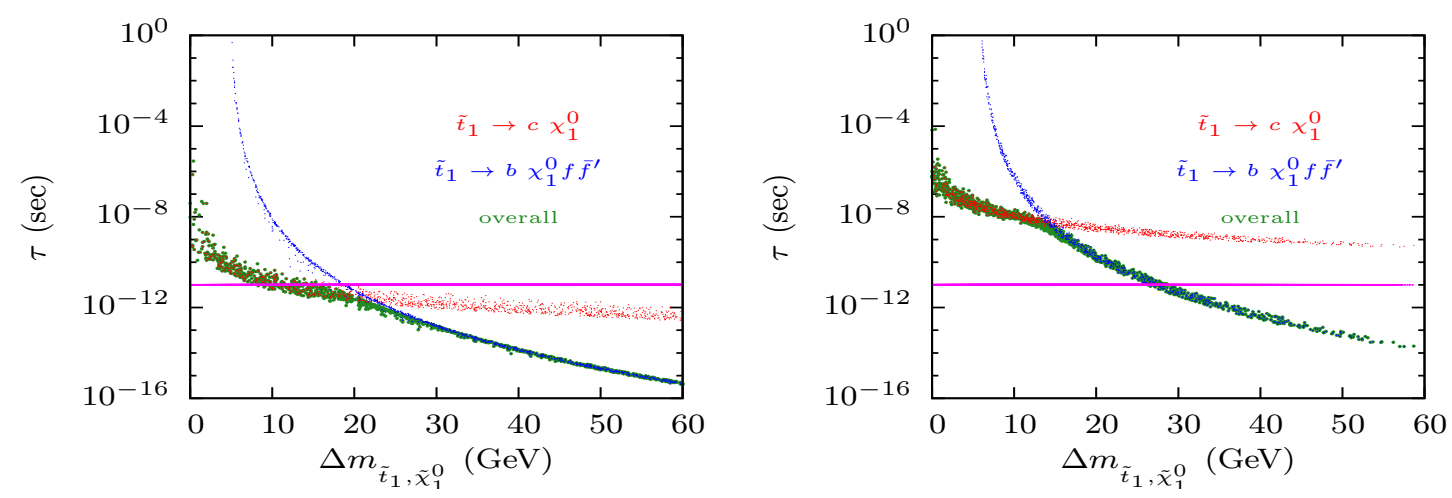

Figure 9. Decay life-time of $\tilde{t}_{1}$ (at rest) if only the two-body $c \tilde{\chi}_{1}^{0}$ mode (in red) or only the fourbody $b f \bar{f}^{\prime} \tilde{\chi}_{1}^{0}$ mode (in blue) were possible as functions of the mass-split $\left(\Delta m_{\tilde{t}_{1}, \tilde{\chi}_{1}^{0}}\right)$ between $\tilde{t}_{1}$ and $\tilde{\chi}_{1}^{0}$. Points in green indicate the values of the actual lifetime of $\tilde{t}_{1}$ (at rest) and result from summing up its decay widths in the two modes. The left plot is for the case of bino-dominated LSP while the right one stands for the case of the singlino-dominated LSP. The scattered points are obtained by scanning over the NMSSM parameter space. See text for details. 

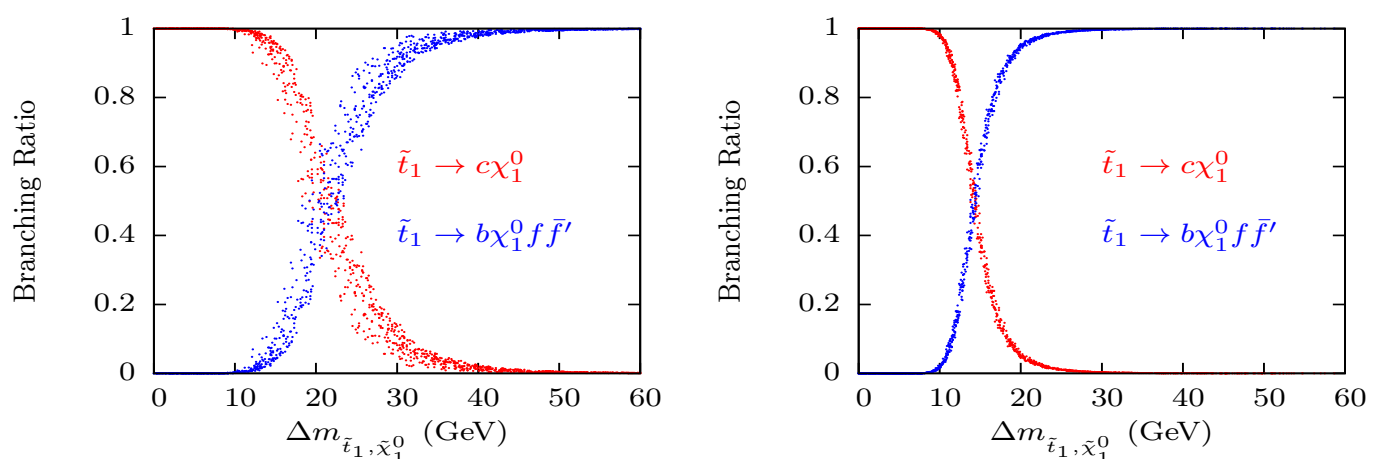

Figure 10. Same as in the case of figure 9 but presenting the respective branching fractions of $\tilde{t}_{1}$.

that while the FV decay width is calculated by NMSSMTools, the $4 \mathrm{~B}$ ones are not. Hence we use MadGraph5_aMC@NLO v2.1.2 [118] to calculate the latter and then get them crosschecked in CalcHEP (v3.6.23) [119]. For the present purpose, the scans are done around the respective benchmark points with $\left|A_{t}\right| \leq 300 \mathrm{GeV}$ and $700 \mathrm{GeV} \leq m_{\tilde{Q}_{3}}, m_{\tilde{U}_{3}} \leq 1 \mathrm{TeV}$, keeping all remaining parameters fixed. For larger mass-splits $\left(\Delta m_{\tilde{t}_{1}, \tilde{\chi}_{1}^{0}}\right)$ between $\tilde{t}_{1}$ and the LSP, the four-body decay dominates. Hence the green bands overlap with the blue bands. For smaller values of $\Delta m_{\tilde{t}_{1}, \tilde{\chi}_{1}^{0}}$, the bottom quark mass $(\sim 5 \mathrm{GeV})$ stands in the way for the four-body decay to dominate. Thus, the FV decay becomes important and the green bands superpose on the red bands in this regime. The scattered points are the results of scans over the NMSSM parameter space. The horizontal lines in the two plots represent a conservatively chosen critical lifetime of $10^{-11}$ second in the rest frame of the decaying $\tilde{t}_{1} \cdot{ }^{12}$ This corresponds to a decay-length (given by $\beta \gamma c \tau$ ) of a few millimeters and can be considered to be prompt for a new physics excitation. In any case, note that for $\Delta m_{\tilde{t}_{1}, \tilde{\chi}_{1}^{0}} \gtrsim 25 \mathrm{GeV}$, which is the case in our present analysis, the overall lifetime is smaller than this critical value. Thus, we are always in the regime where $\tilde{t}_{1}$ undergoes prompt decays irrespective of whether the LSP is bino- or singlino-dominated.

In figure 10 we illustrate the corresponding variations of the branching fractions in the FV and 4B modes for the bino- (left) and the singlino-dominated (right) LSP. Clearly, for $\Delta m_{\tilde{t}_{1}, \tilde{\chi}_{1}^{0}} \gtrsim 20 \mathrm{GeV}$ which is what we have for our benchmark scenarios, the four-body decay takes over. This substantiates the recent appreciation of the importance of the four-body decay of $\tilde{t}_{1}$. Furthermore, an early onset (for a smaller $\Delta m_{\tilde{t}_{1}, \tilde{\chi}_{1}^{0}}$ ) of domination for the four-body branching fraction does not escape notice. This is expected since the FV decay experiences simultaneous suppressions from the loop-process and the diminished coupling for a singlino-like LSP.

\subsection{Signatures at the LHC}

From table 4 it is clear that charm and/or bottom quark(s) in the final states are unavoidable. The sources of the bottom quarks are the two-body decays $\tilde{b}_{1} \rightarrow b \tilde{\chi}_{1}^{0}$ and the

\footnotetext{
${ }^{12}$ For simplicity, we skip the boost factor $\beta \gamma$ where the $\beta=\frac{v}{c}$ and $\gamma=\left(1-\frac{v^{2}}{c^{2}}\right)^{-\frac{1}{2}}$. At the LHC, $\beta \gamma$ has a distribution and may result in an increase of the $\tilde{t}_{1}$ lifetime by a factor of 1.5 to 2 [115].
} 
four-body decays of the $\tilde{t}_{1}$. If the LSP is singlino-like, the former decay is disfavored and the cascades end with decays of $\tilde{t}_{1} / \tilde{t}_{1}^{*}$.

Thus, identifying the $c$ - and the $b$-jets would definitely help probe the scenario [120]. As for $c$-tagging, it is understood to be a dedicated technique with low to moderate efficiencies. This is true, in particular, for charm quarks coming from the decays of the stops having a small mass-split with the LSP. Therefore, we would not demand tagging of $c$-jet(s), although, if becomes possible, this could be rather helpful. ${ }^{13}$ Note that although the scenario is of a compressed kind, in the present case, the charm quarks are not coming from stops that are directly produced in $p p$ collisions. Rather, these are coming from the decays of heavier states like $\tilde{t}_{2}$ and/or $\tilde{b}_{1}$. Thus, the $\tilde{t}_{1} \tilde{t}_{1}^{*}$ system would not be back-to-back (in the plane transverse to the beam axis) unlike when these are directly produced. Such a production of $\tilde{t}_{1} \tilde{t}_{1}^{*}$ under cascades of heavier states, thus, to a limited extent, could play the role of a hard jet from the initial state radiation (ISR) against which the $\tilde{t}_{1} \tilde{t}_{1}^{*}$ recoils in case of their direct production. This would help increase the amount of $\mathbb{E}_{T}$ along with the $p_{T}$ of the $c$-jets even without the presence of a hard ISR jet. As for the $b$-jets, a noteworthy aspect of such a scenario is their natural dearth in the final state. The depletion is primarily at the level of their rates (effective branching fractions; see table 2). The depletion in their rates is severe when $\operatorname{BR}\left(\tilde{b}_{1} \rightarrow b \tilde{\chi}_{1}^{0}\right)$ becomes further suppressed, typical to the case with a singlino-dominated LSP. However, to be convinced of their presence, we incorporate $b$-tagging in our analysis.

On the other hand, with as many as three possible SM gauge bosons $\left(Z\right.$ and $\left.W^{ \pm}\right)$that may appear in the cascade of a $\tilde{t}_{2}$-pair, lepton-rich final states would be common. These leptons could naturally serve as the cleaner probes to such a scenario at the future runs of the LHC. Finding the footprints of one or more on-shell $Z$-bosons (via their reconstructions from pairs of OSSF leptons) in conjunction with finding leptons with origins in decays of on-shell $W$-bosons hold the key. Hence we confine our analysis to final states having three to four leptons. At this point, the following few issues pertaining to the scenario under consideration may be noted down:

- when no $b$-jet is available in the final state, finding one or more $c$-jets could be helpful. There are three ways in which $b$-jets could get depleted in our scenario ${ }^{14}$

- when the LSP is singlino-like and hence $\tilde{b}_{1} \rightarrow b \tilde{\chi}_{1}^{0}$ is negligible,

\footnotetext{
${ }^{13} \mathrm{~A}$ recent ATLAS Note [121] discusses a dedicated $c$-tagging algorithm (in reference to the process $\left.p p \rightarrow \tilde{t}_{1} \tilde{t}_{1}^{*} \rightarrow c \tilde{\chi}_{1}^{0} c \tilde{\chi}_{1}^{0}\right)$. The study reveals that a medium/conservative (loose) set of criteria yields on an average $\mathrm{a} \approx 20 \%(\approx 95 \%)$-tagging efficiency. The medium (loose) criterion seems to be optimal when the background is dominated by light- and $b$-quark jets ( $t \bar{t}$ processes). Given the rather preliminary nature of the study and a more complex topology that we are considering for our signal, it makes sense that at this point we talk about the conservative values of the projected efficiency. For a rather soft $p_{T}^{\text {charm }} \lesssim 20 \mathrm{GeV}$ that we consider, the conservative efficiency now ranges between $10-20 \%$ which is a little too small for our present purpose.

${ }^{14}$ It is worthwhile to note that even when we deal with three squarks from the third generation, there are various different possibilities which result in final states with depleted or even zero b-jets. This can be traced back to the fact that in our scenario the lighter chargino is heavier than $\tilde{t}_{2}$ and hence the decay $\tilde{t}_{2} \rightarrow b \tilde{\chi}_{1}^{+}$is closed. Thus, out of the three light squarks, only the decay of $\tilde{b}_{1}$ could lead to a $b$-jet.
} 
- when the LSP is bino-like but $\tilde{t}_{2}$ dominantly decays to $\tilde{t}_{1} Z$ followed by $\tilde{t}_{1} \rightarrow c \tilde{\chi}_{1}^{0}$ and

- when the LSP is again bino-dominated but the decay $\tilde{b}_{1} \rightarrow \tilde{t}_{1} W^{-}$dominates over the decay $\tilde{b}_{1} \rightarrow b \tilde{\chi}_{1}^{0}$.

- $b$-jets always become handy whenever they appear (only favored for a bino-dominated LSP). Also, for our benchmark points the splitting $m_{\tilde{b}_{1}}-m_{\tilde{\chi}_{1}^{0}}$ is around $100 \mathrm{GeV}$ thus making the $b$-tagging efficiency near-maximal for those $b$-s that come from the decays of $\tilde{b}_{1}$. The $b$-quarks having origins in the four-body decays of $\tilde{t}_{1} / \tilde{t}_{1}^{*}$ are presumably too soft to be efficiently detected in the present setup.

- A naturally 'charm-less' final state is possible (see the fourth entry $\left((c)\left(c^{*}\right)\right)$ of table 4$)$ when tagging of the b-jets would be all the more important. The $\tilde{t}_{2}$-cascade that leads to this channel (see figure 8c) involves a $W$-boson. Thus, there can be up to two leptons in a charm-less final state.

- Charm-less final states are also possible when both $\tilde{t}_{1}$ states decay via the fourbody mode (see, for example, the first entry of table 4 (mode B)). Leptons that are presumably too soft to be easily detected may appear in such decays.

- It may also be noted that such four-body decays of $\tilde{t}_{1} / \tilde{t}_{1}^{*}$ could result in $c$-quarks (via off-shell $W$-bosons leading to the ' $c s$ ' final state (see entry $\left((a)\left(a^{*}\right)\right)$ of table 4 , mode C), which might have an even softer $p_{T}$ spectrum when compared to the same for the $c$-quark arising from the decay $\tilde{t}_{1} \rightarrow c \tilde{\chi}_{1}^{0}$.

- If detectable, final states with various possible combinations of $c$ - and $b$-jet multiplicities could turn out to be efficient and corroborative probes for an in-depth understanding of such a scenario.

To define the optimal signal regions, following the above discussions, we first pick up five appropriate final states. These are listed in table 5 . The second column presents the actual search modes and these can be directly traced back to table 4 and thus, in turn, to the contributing cascades of $\tilde{t}_{2}$ illustrated in figure 8. In the last column of table 5 we indicate the sources of dominant SM backgrounds against each of the signal final states. It is to be noted that bare $t \bar{t}$ production, by itself, is not a dominant background for the multilepton $\left(n_{\ell} \geq 3\right)$ final states under consideration except for the SSDL one. The choice of these final states are prompted by their cleanliness due to the presence of three or more leptons. These leptons have their origins in the $Z$ - and the $W$-bosons appearing in the cascades of $\tilde{t}_{2}$. Hence at least one pair of opposite sign same flavor (OSSF) (with a maximum of two such pairs) can be reconstructed to the mass of the $Z$-boson.

An exception to this is the final state with a lower lepton multiplicity in the form of same-sign dileptons (SSDL). This final state is traditionally known to be extremely clean given that the SM background is naturally suppressed. In our scenario, such a final state (SRSSDL0b) is a possibility when $\tilde{t}_{2} \tilde{t}_{2}^{*}$ pair undergoes the cascade indicated by figure $8(\mathrm{~b})$ (and the corresponding entry in table 3). More specifically, an SSDL final state arises from 


\begin{tabular}{|c|c|c|}
\hline $\begin{array}{l}\text { Channel } \\
\text { ID }\end{array}$ & Search channel & $\begin{array}{c}\text { Dominant } \\
\text { backgrounds }\end{array}$ \\
\hline SRSSDL0b & $\mathrm{SSDL}+(4,6) j+0 b+\not{E}_{T}$ & $t \bar{t}, t \bar{t} W, t \bar{t} Z, Z W$ \\
\hline SRSSDL1b & $\mathrm{SSDL}+(3,4) j(1 b-j e t)+\mathbb{E}_{T}$ & $t \bar{t}, t \bar{t} W, t \bar{t} Z, Z W$ \\
\hline SR3 $30 \mathrm{~b}$ & $3 \ell+(3,4) j+0 b+\mathbb{E}_{T}$ & $Z W, t \bar{t}, t \bar{t} Z, Z Z W$ \\
\hline SR3 $3 \ell 1 b$ & $3 \ell+\leq 2 j(1 b-j e t)+\mathbb{E}_{T}$ & $t \bar{t} Z, t \bar{t}, t \bar{t} W, Z W$ \\
\hline 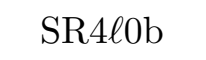 & $4 \ell+(1,2) j+0 b+\mathbb{E}_{T}$ & $Z Z, Z W W, Z Z W$ \\
\hline
\end{tabular}

Table 5. Definition of the signal regions in terms of the actual search channels undertaken in the present analysis. Leptons have their origins in the $Z$ - and the $W$-bosons appearing in the cascades of $\tilde{t}_{2}$. At least one pair of leptons (opposite sign, same flavor (OSSF); electrons and muons only) is to come from an on-shell $Z$-boson. "SSDL" in the first two rows stands for same-sign dilepton final state. The last column presents the dominant SM background processes corresponding to each final state. These are inclusive of two hard jets except for the $Z W$ and $t \bar{t}$ processes for which three-jet inclusive samples are used.

the leptonic decays of those $W$-bosons having the same electric charge that appear at two different stages of the cascades of $\tilde{t}_{2}$ and $\tilde{t}_{2}^{*}$.

Note that in the scenario we consider, the charm and the bottom quark jets coming from the decays of $\tilde{t}_{1} / \tilde{t}_{1}^{*}$ would be much softer with $p_{T}^{\text {jet }} \lesssim 50 \mathrm{GeV}$. Hence there is a fair chance of losing such reconstructed jets at the detectors. Furthermore, as pointed out earlier, the final states are not generally rich in harder $b$-jets. In our analysis, we tag the $b$-jets and accept or veto them as per requirement. As we will discuss shortly, the background to final state(s) with a $b$-jet mainly arises from the generic processes like $t \bar{t}+j e t s$. However, the requirement of a large lepton-multiplicity effectively reduces this background. On the other hand, the final states without a $b$-jet, in addition, draw backgrounds from the SM processes like vector-boson pairs plus extra jets. Demanding a minimum $\mathbb{E}_{T}$ could help tame these backgrounds. As for the SM backgrounds, we consider three-jet inclusive samples for the $Z W$ (which can give rise to trileptons) and $t \bar{t}$ processes. For other background processes, we only consider samples inclusive of two extra hard jets.

\subsection{Simulation}

The exclusive signal process considered for our analysis is the basic production of a pair of $\tilde{t}_{2}$ at the LHC $\left(p p \rightarrow \tilde{t}_{2} \tilde{t}_{2}^{*}\right)$ followed by their cascades. The background processes we consider are already listed in the previous subsection. For both signal and the background processes, event samples are generated at the lowest order (LO) in perturbation theory using MadGraph5_aMC@NLO v2.1.2 [118]. CTEQ6L1 [122] parton distribution function is used with the factorization/renormalization scale set at the default MadGraph setting (i.e., at $m_{T_{\tilde{t}_{2}}}=\sqrt{m_{\tilde{t}_{2}}^{2}+p_{T_{\tilde{t}_{2}}}^{2}}$ ).

For the signal, the next-to-leading-order (NLO) plus next-to-leading-log (NLL) cross sections are computed with the package NLL-fast (v3.0) [123] which in turn uses the package Prospino2 (v2.1) [124] for the NLO result. CTEQ6.6M parton distribu- 


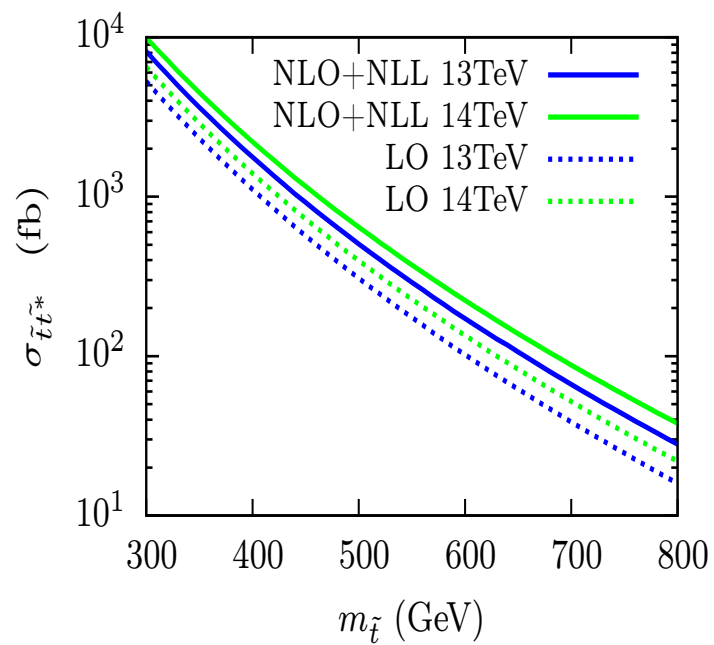

Figure 11. Production cross sections (in femtobarn) of a pair of stops as functions of the stop mass at LHC-13 and LHC-14. The lower two curves correspond to the LO estimations while the upper ones include NLO+NLL effects.

tion parametrization, default to the package, has been employed for the purpose. The NLO+NLL cross sections are later used to normalize the signal rate. In figure 11 we illustrate the variation of the $\tilde{t}_{2} \tilde{t}_{2}^{*}$ production cross section at LHC-13 and LHC-14 without and with the NLO+NLL correction as functions of $\tilde{t}_{2}$ mass. On the other hand, to be on the conservative side, we apply $K$-factors of 1.6 for the backgrounds from $t \bar{t}$ processes (with inclusive jets) and 1.3 for all the others.

Events generated for both the signal and the backgrounds are then showered and the unstable particles are decayed using Pythia (v6.426) [125] embedded within the Madgraph environment. For the signal, the decay branching fractions of various SUSY excitations calculated within the framework of NMSSMTools [60] are provided to Pythia through the SLHA2 [126] interface. ${ }^{15}$ To avoid double counting of events in the background samples in the presence of extra hard partonic jets and the parton shower, we employ the MLM matching scheme [127-129] with the variables xqcut and qcut set at appropriate values.

\subsubsection{Reconstructing the physics objects}

The fast detector simulation framework DELPHES (v3.1.2) [130] is used to reconstruct final physics objects. The following steps are taken to obtain them in an ATLAS environment (in terms of the detector coverage).

- The jet-finding package Fast Jet (v3.0.6) [131] embedded in DELPHES is used to find the jets. The anti- $k_{T}$ jet algorithm is employed with the cone size set at 0.4 , requiring a minimum $p_{T}^{\text {jet }}$ of $20 \mathrm{GeV}$ and the pseudorapidity in the range $\left|\eta_{\text {jet }}\right|<2.5$. A flat $b$-tag efficiency of $70 \%$ is incorporated. Furthermore, we consider a $20 \%$ probability

\footnotetext{
${ }^{15}$ The four-body decay width obtained from Madgraph (see section 4.1) is inserted appropriately into the SLHA2 file generated by NMSSMTools which contains all other decay information. This ensures correct estimation of $\tilde{t}_{1}$ branching fractions.
} 
of a $c$-jet being mistagged as a $b$-jet while the same with the light quark flavors are taken to be $1 \%$ [120, 132].

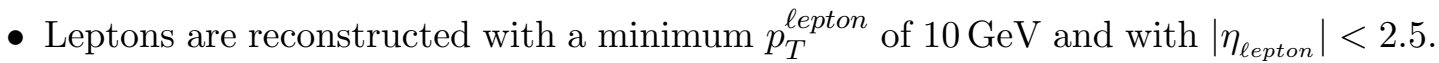
For electrons and muons, we remove jets which lie within an angular distance $\Delta R \leq$ 0.2 from the lepton. Also, to increase the purity of electrons, it is required that the ratio of total $p_{T}$ 's of the stray tracks within the cones of their identification to their own $p_{T}$ 's is less that 0.1 . The corresponding requirement for the muons is that the maximum total $p_{T}$ of other tracks does not exceed $1.8 \mathrm{GeV}$.

The entire process of event-analysis is done within the modular and integrated framework of the popular package of MadAnalysis 5 (v1.1.11) [133,134] with the help of the in-built data analysis framework ROOT (v5.34.22) [135] using the event format STDHEP [136].

\subsubsection{Event selection}

In table 6 we list three sets of kinematic cuts that are employed in our analysis for five

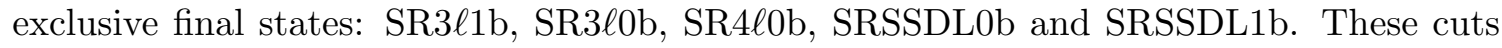
remain the same for the corresponding (jet-)inclusive samples as well (which we discuss at length) except for lifting the jet-related cuts (jet $p_{T}$, jet-multiplicity etc.). However, these sets are common across all the three benchmark points that we consider and for both LHC-13 and LHC-14. Graded cuts are applied on the $p_{T}$ 's of leptons and jets and on the transverse masses (defined as $m_{T}=\sqrt{2 p_{T}^{\ell} \mathbb{E}_{T}\left[1-\cos \left(\phi_{\ell}-\phi_{\mathbb{E}_{T}}\right)\right]}$ ) of the leptons based on their relative hardness. For this analysis, by leptons we mean electrons and muons. It may be noted that one cannot afford a more stringent lower cut on $p_{T}$ of the jets. This is because, unlike in generic SUSY searches, even involving squarks from the third generation, where jets may arise either directly from rather heavy colored states or in the decays of heavy electroweak gauginos, the sources of jets in the present case are only the $W / W^{*}$ bosons. On top of that, we work with not only light spectra but also somewhat compressed ones which further deplete the $p_{T}$ of the jets.

For the same reason, one can expect only moderately hard $\mathbb{E}_{T}$ for any of the targeted final states and for any of the benchmark points. This is apparent from figure 12. Therein we plot the $\mathscr{E}_{T}$ distributions (left) and the effective mass $\left(m_{\mathrm{eff}}=\sum_{i} p_{T_{i}}^{\text {visible }}+\mathbb{E}_{T}{ }{ }^{\prime}{ }^{\prime} i\right.$ ' standing for the $i$-th visible state; right) for the SM background (in red) and for the three benchmark points (for inclusive final states) for LHC-13. The $\mathbb{E}_{T}$ spectra peaks at around $100 \mathrm{GeV}$ irrespective of the benchmark scenario. Note that this peak value is already on the smaller side given the mass of the LSP is around $300 \mathrm{GeV}$. Consequently, one finds degraded peaks in the effective mass distributions at around $400 \mathrm{GeV}$ which characterize a compressed spectrum, while under normal circumstances, a flatter peak about the total mass $(\sim 1 \mathrm{TeV})$ of the pair-produced particles $\left(\tilde{t}_{2}\right)$ is expected. We do not impose any separate cut on $m_{\mathrm{eff}}$ as this does not improve the situation much once a cut on $\mathbb{E}_{T}$ is imposed.

In figure 13, from left to right, we illustrate the $m_{T}$ distributions of the three hardest leptons $\ell_{1}, \ell_{2}$ and $\ell_{3}$ with $p_{T}^{\ell(1)}>p_{T}^{\ell(2)}>p_{T}^{\ell(3)}$ for the SM background (in red) and for the signal benchmark point BP1 (in blue) in the inclusive multilepton final state at LHC13. We do not find any major discriminatory feature among the $m_{T}$ distributions of the 


\begin{tabular}{|c|c|c|c|c|c|}
\hline Variables & SR $3 \ell 1 b$ & SR3 $30 \mathrm{~b}$ & 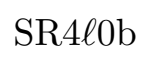 & SRSSDL0b & SRSSDL1b \\
\hline$n_{\ell}$ & 3 & 3 & 4 & 2 (SSDL) & $2(\mathrm{SSDL})$ \\
\hline$n_{\ell \ell}^{\mathrm{OSSF}}$ & 1 & 1 & 2 & vetoed & vetoed \\
\hline$M_{\ell \ell}^{\mathrm{OSSF}}(\mathrm{GeV})$ & \multicolumn{3}{|c|}{$75 \leq M_{\ell \ell}^{\mathrm{OSSF}} \leq 105$} & - & - \\
\hline $\begin{array}{c}n_{j e t} \\
n_{b-j e t} \text { (incl.) }\end{array}$ & $\begin{array}{c}1 \text { to } 2 \\
1\end{array}$ & $\begin{array}{l}3 \text { to } 4 \\
\text { vetoed }\end{array}$ & $\begin{array}{l}1 \text { to } 2 \\
\text { vetoed }\end{array}$ & $\begin{array}{l}4 \text { to } 6 \\
\text { vetoed }\end{array}$ & $\begin{array}{c}3 \text { to } 4 \\
1\end{array}$ \\
\hline $\begin{array}{c}p_{T}^{j_{(n)}}(\mathrm{GeV}) \\
p_{T}^{b-j e t}(\mathrm{GeV})\end{array}$ & \multicolumn{5}{|c|}{$\begin{array}{r}p_{T}^{j_{(1,2,3, \geq 4)}}>(30,30,25,20) \\
p_{T}^{b-j e t}>40(\text { wheneve }\end{array}$} \\
\hline$p_{T}^{\ell}(\mathrm{GeV})$ & \multicolumn{5}{|c|}{$p_{T}^{\ell(1,2,3,4)}>(25,20,20,15)$} \\
\hline$m_{T}^{\ell_{(n)}}(\mathrm{GeV})$ & \multicolumn{5}{|c|}{$\begin{array}{c}m_{T}^{\ell_{(1,2,3)}}>(140,125,100) \\
\text { (not applicable for SR4L0b) }\end{array}$} \\
\hline $\mathbb{E}_{T}(\mathrm{GeV})$ & \multicolumn{5}{|c|}{$\mathbb{E}_{T}>135$} \\
\hline
\end{tabular}

Table 6. The complete definition of the signal regions (SR) denoting the final states they represent and the respective sets of common selection cuts on the physics objects that are independent of the benchmark scenarios and the LHC energies. By leptons only electron and muon are referred to. Other notations follow the standard conventions.

leptons in different final states and for LHC-14. Hence we stick to a common set of selection criteria for them as indicated in table 6. Note that for the 4-lepton final state (SR $4 \ell 0 \mathrm{~b}$ ), the leptons come from a pair of on-shell $Z$-bosons. Hence we do not impose the $m_{T}$-cuts for this final state.

For all the three variables we discussed, we do not separately present plots for LHC14 as they are rather similar (except for, as expected, the tails getting extended slightly towards the harder side).

\subsubsection{Results and discussions}

In this subsection we discuss the results of our simulations for LHC-13 and LHC-14, and for different signal regions described in tables 5 and 6 . The purpose of the simulation is to understand how sensitive the LHC experiments are to the proposed NMSSM scenario with two relatively light stops.

It is important to note that the data $\left(\sim 20 \mathrm{fb}^{-1}\right.$ for each of ATLAS and CMS $)$ from LHC-8 are not at all sensitive to the scenario we propose. This is in spite of rather low stop masses with $m_{\tilde{t}_{1}} \gtrsim 300 \mathrm{GeV}$ and $m_{\tilde{t}_{2}} \lesssim 500 \mathrm{GeV}$ that we choose in this work. This can be understood in the following way. First, our scenario is somewhat of a compressed kind where the mass-split between the NLSP $\tilde{t}_{1}$ and the LSP $\left(\tilde{\chi}_{1}^{0}\right)$ does not exceed $\sim 30 \mathrm{GeV}$. Thus, $\tilde{t}_{1}$ decays can decay only to the FV mode $c \tilde{\chi}_{1}^{0}$ and the four-body $b f \bar{f}^{\prime} \tilde{\chi}_{1}^{0}$ mode. We respect the relevant bound which is $m_{\tilde{t}_{1}} \gtrsim 250 \mathrm{GeV}[89,90]$ and is grossly applicable to our 

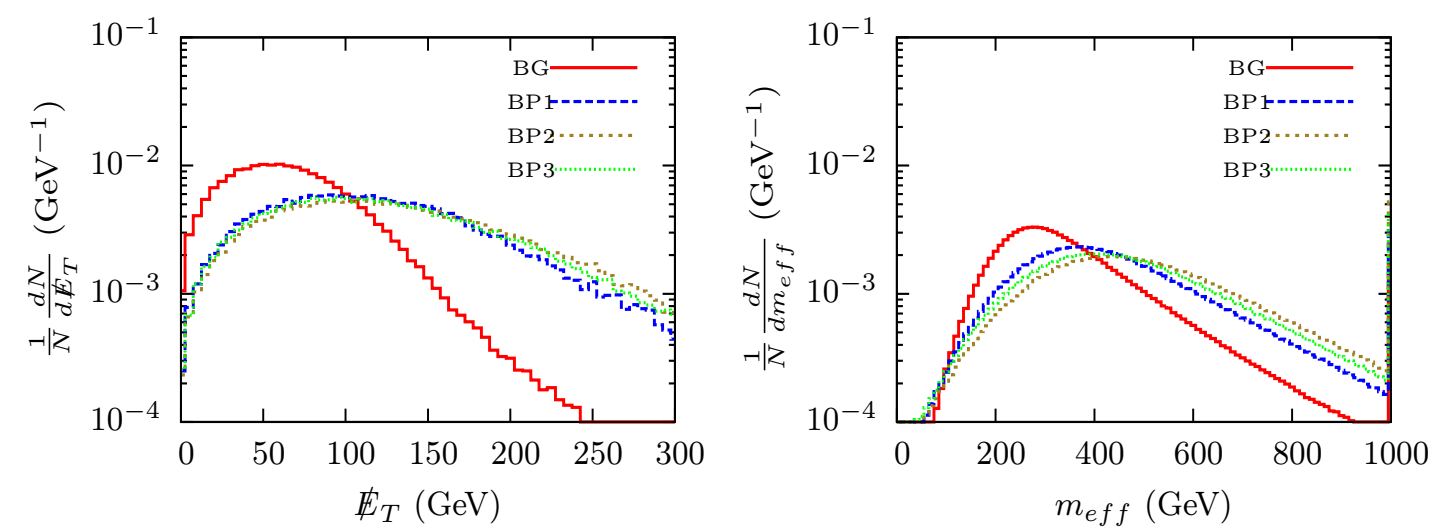

Figure 12. $\mathbb{E}_{T}$ distributions (left) and $m_{\text {eff }}$ distributions (right) for the SM background (in red) and for the signal in the inclusive multilepton final state for the three benchmark points (in blue, brown and green, respectively) and for LHC-13.
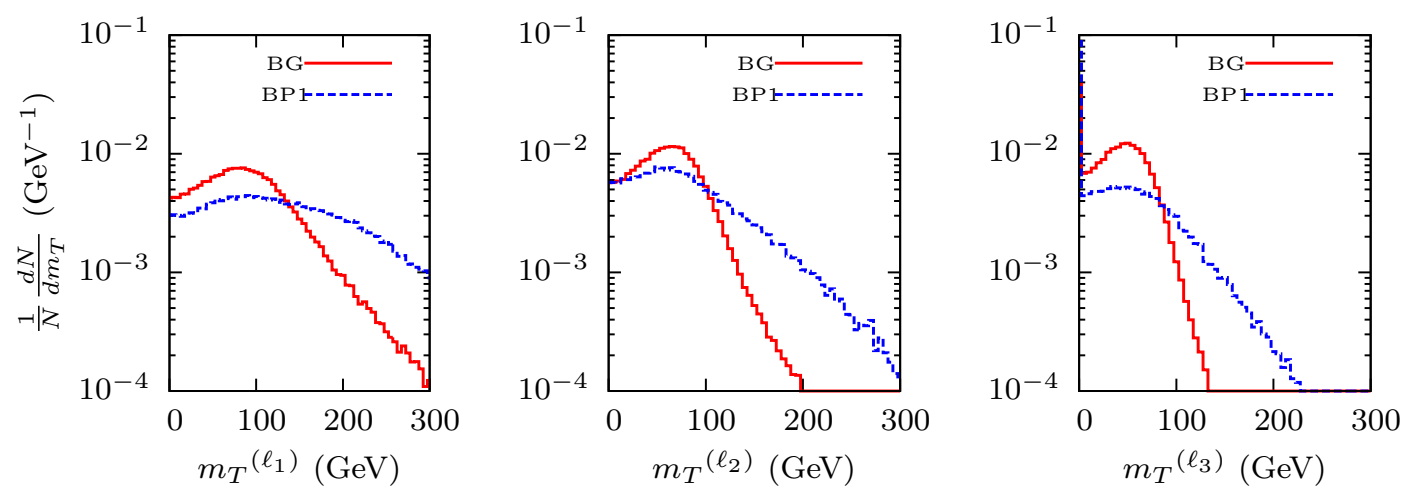

Figure 13. $m_{T}$ distributions of the three hardest leptons $\ell_{1}, \ell_{2}$ and $\ell_{3}$ (in order of decreasing hardness; from left to right) for the SM background (in red) and for the signal benchmark point BP1 (in blue) at LHC-13 and in an inclusive sample.

scenario (except under specific situations when the LSP is singlino dominated and thus $\tilde{t}_{1}$ could become relatively long-lived at the collider). Our choice of $m_{\tilde{t}_{1}} \approx 300 \mathrm{GeV}$ is thus conservative to a good extent and it may be only natural to expect that LHC-8 would not be sensitive to such a stop squark.

However, the scenario is not all about the lighter stop on its own. In fact, the combined phenomenology of the two stops together is what defines the situation. Note that in terms of the basic production mechanism, the phenomenology is entirely governed by the pairproduction rate of $\tilde{t}_{2}$. However, the final state may or may not involve a decaying $\tilde{t}_{1}$ (see tables 3 and 4). While all usual mass bounds obtained at colliders for $\tilde{t}_{1}$ is generic for a stop-like state having the same set of decay modes and hence applicable to the heavier stop as well, a more practical and stringent sets of constraints respecting their hierarchy have recently been obtained by the LHC experiments $[84,85] .{ }^{16}$ Whatever the case may be, these analyses are again expected to have enhanced sensitivity thanks to the assumptions that

\footnotetext{
${ }^{16}$ An interesting caveat of these studies may be that these are silent about the decay branching fraction $\tilde{t}_{2} \rightarrow t \tilde{\chi}_{1}^{0}$. This is unavoidable when they assume $\tilde{t}_{1} \rightarrow t \tilde{\chi}_{1}^{0}$ is $100 \%$. It would be interesting to see in what way $\operatorname{BR}\left[\tilde{t}_{2} \rightarrow t \tilde{\chi}_{1}^{0}\right]$ affects these analyses.
} 
$\tilde{t}_{1} \rightarrow t \tilde{\chi}_{1}^{0}$ is possible and has a $100 \%$ branching fraction. This is consistent with the fact that more stringent bounds are obtained from $\tilde{t}_{1}$ searches at the LHC with such an assumption when compared to those obtained in studies assuming $\tilde{t}_{1} \rightarrow c \tilde{\chi}_{1}^{0}$ and $\tilde{t}_{1} \rightarrow b f \bar{f}^{\prime} \tilde{\chi}_{1}^{0}$ as the only possibilities.

We now turn to the case of imminent LHC-13 followed by a possible case of LHC-14 in the near future. In table 7 we present yields for the backgrounds in various different final states and the corresponding numbers for the signal events accompanied by the signal significance (in parentheses) in each case for an accumulated luminosity of $100 \mathrm{fb}^{-1}$. These are done for all the three benchmark points and for both LHC-13 and LHC-14. Kinematic cuts as presented in table 6 are used in the analysis. The significances are estimated using the expression (see appendix A of [137])

$$
\sigma=\sqrt{2\left[(S+B) \ln \left(1+\frac{S}{B}\right)-S\right]}
$$

which is applicable to situations with small number of events (in particular, when the number of background events is less than 50), is based on likelihood-ratios and follows from the Poisson distribution.

As can be seen from table 7 , none of the modes except for the 4-lepton final state

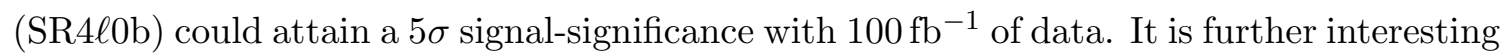
to note that for SR $4 \ell 0 \mathrm{~b}, \mathrm{a} \sim 5 \sigma$ significance is achievable for scenarios with a singlino-like LSP (BP3) or with one having a bino-like LSP (BP2) for which $\tilde{b}_{1}$ mostly decays to $\tilde{t}_{1} W$, similar to as it does in the case of BP3. Note that the final state SR $3 \ell 1 \mathrm{~b}$, for the benchmark points BP2 and BP3, has the poorest strength of all reaching barely the $1 \sigma$ level. This is not surprising since SR3 $\ell 1 b$ requires a tagged $b$-jet which, in our scenario, would mostly come from the decay $\tilde{b}_{1} \rightarrow b \tilde{\chi}_{1}^{0}$. But this branching fraction is suppressed in BP2 and BP3 (see table 2). Same is the explanation for a poor show of the final state SRSSDL1b for BP2 and BP3. In any case, it thus appears that these final states, which are somewhat of an exclusive kind as far as the number of jets (including the b-jets) associated with each of them are concerned (see table 6), are not very sensitive even to a moderately large volume of data at the imminent run(s) of the LHC.

The situation prompts us to explore an alternative strategy. It is found that looking for jet-inclusive final states for all these multi-lepton modes holds a better prospect. In table 8 we present the corresponding numbers but after lifting the jet-related cuts (on multiplicity and $p_{T}$ ). It is clear that a $\gtrsim 4 \sigma$ significance is possible for final states with larger (3 to 4) lepton multiplicity. For the benchmarks BP2 and BP3, signals in the inclusive 3- and 4-lepton final states could even attain a significance of up to $6-7 \sigma$. It is also to be pointed out that as far as the LHC phenomenology is concerned, the possibility of a large $(\sim 1)$ $\operatorname{BR}\left[\tilde{b}_{1} \rightarrow b \tilde{\chi}_{1}^{0}\right]$ in the bino-dominated LSP makes all the difference. This is the case with the benchmark point BP1. It is clearly seen from table 7 that the signal rates and their mutual patterns for BP1 are very different from those for BP2 and BP3. At the same time, the latter two, though represent different scenarios, have similar branching patterns for the sbottom and the stop squarks (see table 2) which result in pretty similar rates and patterns for various final states. Furthermore, the usual expectation is that the significance for 


\begin{tabular}{|c|c|c|c|c|c|}
\hline \multirow{2}{*}{$\begin{array}{c}\text { Channel } \\
\text { ID }\end{array}$} & \multirow{2}{*}{$\begin{array}{c}\sqrt{s} \\
(\text { in } \mathrm{TeV})\end{array}$} & \multicolumn{4}{|c|}{ Number of events and signal significances } \\
\hline & & Background & $\mathrm{BP} 1(\sigma)$ & $\mathrm{BP} 2(\sigma)$ & $\mathrm{BP} 3(\sigma)$ \\
\hline \multirow{2}{*}{ SRSSDL0b } & 13 & 9.7 & $1.8(0.6)$ & $7.3(2.1)$ & $8.6(2.5)$ \\
\hline & 14 & 20.0 & $2.6(0.6)$ & $10.9(2.3)$ & $11.0(2.3)$ \\
\hline \multirow[t]{2}{*}{ SRSSDL1b } & 13 & 20.3 & $2.3(0.5)$ & $1.9(0.4)$ & $1.4(0.3)$ \\
\hline & 14 & 26.1 & $2.4(0.5)$ & $2.4(0.5)$ & $1.9(0.4)$ \\
\hline \multirow[t]{2}{*}{ SR3€0b } & 13 & 12.1 & $4.1(1.1)$ & $11.3(2.9)$ & $11.5(2.9)$ \\
\hline & 14 & 20.0 & $5.1(1.1)$ & $15.6(3.1)$ & $15.5(3.1)$ \\
\hline \multirow[t]{2}{*}{$\mathrm{SR} 3 \ell 1 \mathrm{~b}$} & 13 & 8.0 & $6.2(2.0)$ & $2.6(0.9)$ & $2.0(0.7)$ \\
\hline & 14 & 5.4 & $7.9(2.9)$ & $3.4(1.3)$ & $2.4(1.0)$ \\
\hline \multirow[t]{2}{*}{ SR $4 \ell 0 \mathrm{~b}$} & 13 & 1.7 & $5.4(3.1)$ & $8.7(4.5)$ & $9.8(5.0)$ \\
\hline & 14 & 3.5 & $7.0(3.0)$ & $11.0(4.4)$ & $12.8(4.9)$ \\
\hline
\end{tabular}

Table 7. Number of background events in different final states and the same for the signal events for the three benchmark points after cuts at LHC-13 and LHC-14. $\sigma$ in the parentheses corresponds to the significance level at an integrated luminosity of $100 \mathrm{fb}^{-1}$.

\begin{tabular}{|c|c|c|c|c|c|}
\hline \multirow{2}{*}{$\begin{array}{c}\text { Jet-inclusive } \\
\text { final states }\end{array}$} & $\sqrt{s}$ & \multicolumn{4}{|c|}{ Number of events and signal significances } \\
\cline { 3 - 6 } & in TeV) & Background & BP1 $(\sigma)$ & BP2 $(\sigma)$ & BP3 $(\sigma)$ \\
\hline \multirow{2}{*}{ SRSSDL } & 13 & 175.9 & $18.2(1.4)$ & $28.5(2.1)$ & $29.6(2.2)$ \\
\cline { 2 - 6 } & 14 & 203.1 & $22.1(1.5)$ & $38.3(2.6)$ & $39.1(2.7)$ \\
\hline \multirow{2}{*}{ SR3 $\ell$} & 13 & 84.1 & $42.0(4.3)$ & $60.8(6.0)$ & $63.7(6.3)$ \\
\cline { 2 - 6 } & 14 & 102.2 & $52.7(4.8)$ & $78.2(7.0)$ & $79.5(7.1)$ \\
\hline \multirow{2}{*}{ SR4 $\ell$} & 13 & 12.1 & $15.2(3.7)$ & $23.3(5.4)$ & $25.7(5.9)$ \\
\cline { 2 - 6 } & 14 & 21.0 & $20.5(3.9)$ & $30.6(5.6)$ & $33.3(6.0)$ \\
\hline
\end{tabular}

Table 8. Same as in table 7 but for jet-inclusive final states. See text for details.

\begin{tabular}{|c|c|c|c|}
\hline \multirow{2}{*}{$\begin{array}{c}\text { Jet-inclusive } \\
\text { final states }\end{array}$} & \multicolumn{3}{|c|}{ Target Luminosity $\left(\mathrm{fb}^{-1}\right)$} \\
\cline { 2 - 4 } SRSSDL & 1000 & 350 & BP3 \\
\hline SR3 & 100 & 50 & 550 \\
SR4 $\ell$ & 150 & 80 & 70 \\
\hline
\end{tabular}

Table 9. Ballpark target (integrated) luminosities (in $\mathrm{fb}^{-1}$ ) required at LHC-13/14 to obtain a $5 \sigma$ signal significance in various final states and for different benchmark points. 
new physics signal with rather massive states would go up as the collision energy grows. In other words, the reach in mass is expected to increase with increasing energy of the experiment. Table 8 indeed shows such an increase in the significance (for all the final states) with increasing energy. However, the numbers show that LHC-13 could be already efficient enough and simply pushing the machine energy to $14 \mathrm{TeV}$ may not be particularly helpful, at least in the present context.

The overall sensitivity and/or reach of these experiments to the scenario in context are best demonstrated by the projected target (integrated) luminosities. In table 9 we

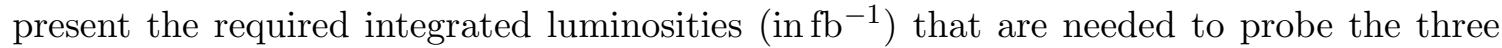
benchmark scenarios at different signal regions. As discussed in the last paragraph, given that they are expected to be rather similar for LHC-13 and LHC-14, we only present a single ballpark value of the target luminosity for each case. The results can be summarized by saying that the simplified scenario we propose with two light stops are most sensitive to final states with large (3 to 4 ) lepton multiplicity. The luminosity requirements for such modes in BP2 and BP3 reflect that these benchmark spectra copiously contribute to such multi-lepton final states.

\section{Conclusions}

A light stop is indispensable for a 'natural' SUSY solution to the notorious hierarchy problem. Within the MSSM such a possibility is now under some tension as the observed value of the Higgs mass generically implies somewhat heavy stops. Set against this, we study the viability of not only one but both stops being relatively light (thus helping in resurrecting the 'natural' SUSY) within the framework of vanilla ( $Z_{3}$-symmetric) NMSSM and their implications for the LHC. The ' $\lambda$ ' parameter of the NMSSM, associated with a new interaction term in the superpotential involving a singlet chiral superfield, gives rise to a tree-level contribution to the Higgs mass. This efficiently makes up for the lightness of the stops in such a scenario and liberates the stop sector from the Higgs sector to a pretty good extent. We point out that direct searches at the LHC still allow for such light stops.

We adopt a 'minimal' scenario with a pair of light stops. A light sbottom is then naturally present. The lighter stop is only a few tens of a GeV heavier than the LSP neutralino. Thus, the only allowed decays of $\tilde{t}_{1}$ are its flavor-violating two-body decay to $c \tilde{\chi}_{1}^{0}$ and its four-body decay to $b f \bar{f}^{\prime} \tilde{\chi}_{1}^{0}$. The LSP is also heavy enough to prohibit the decay $\tilde{t}_{2} \rightarrow t \tilde{\chi}_{1}^{0}$. The decays $\tilde{t}_{2} \rightarrow \tilde{t}_{1} Z$ and/or $\tilde{t}_{2} \rightarrow \tilde{b}_{1} W^{+}$are considered to be characteristic of the scenario. The benchmark scenarios studied in the present work have the mass-hierarchy $m_{\tilde{\chi}_{1}^{0}}<m_{\tilde{t}_{1}}<m_{\tilde{b}_{1}}<m_{\tilde{t}_{2}}$. These low-lying states have their masses in the range $\sim(300-$ 500) GeV. Clearly, the spectra are of a 'compressed' kind, with all other SUSY states taken to be heavy enough to get effectively decoupled. We point out that the compositions (singlino/bino contents) of the LSP neutralino could crucially influence the phenomenology of such a scenario.

The viability of such a scenario is demonstrated by subjecting the NMSSM parameter space to all relevant constraints from low energy electroweak data in various different sectors, to the latest constraints from the dark matter sector and on the theoretical side, 
to the condition of absence of Landau poles. Benchmark points are chosen based on the compositions of the LSP and the key decay modes of $\tilde{t}_{2}$ and $\tilde{b}_{1}$. At colliders, such a scenario would lead to leptons, jets and $\mathbb{E}_{T}$ all of which can at best be moderately hard. A priori, this may erode the sensitivity of the LHC experiments to the scenario under consideration.

Possible cascades of the stop and the sbottom squarks are discussed. $\tilde{t}_{1}$ decays promptly for the chosen benchmarks. Fast simulations (by including detector effects) are performed. Lepton-rich $(\geq 3)$ events, with some of the lepton-pairs reconstructible to $Z$ bosons, are found to be rather characteristic of such a scenario. Finding a $b$-jet could be corroborative while tagging of $c$-jets to real benefit appears a bit futuristic. Interestingly, even with three light squarks from the third generation, contrary to common expectations, some prominent final states could well have depleted or no b-jets. SM backgrounds are simulated thoroughly.

It is found that the LHC-8 is not sensitive to the proposed scenario. The study shows that the LHC-13/14 with an accumulated luminosity of a few tens to a few hundreds of an inverse femtobarn would help explore the scenario. In the presence of signals, a multi-channel analysis could offer a quicker and holistic understanding of such a scenario. Furthermore, allowing for a hard ISR jet could help achieve harder final state objects and hence a cleaner signal region. The bottom-line is that such a scenario with two light stops may initially show up in very general final states which are not so typical in the search for stop $\operatorname{squark}(\mathrm{s})$.

Before we close, the following few observations would be in place:

- Existing and any future bounds on the stop mass assuming that it decays only to flavor-violating $c \tilde{\chi}_{1}^{0}$ and four-body $b f \bar{f}^{\prime} \tilde{\chi}_{1}^{0}$ would broadly be applicable to the scenario we consider, irrespective of whether the LSP $\left(\tilde{\chi}_{1}^{0}\right)$ is bino- or singlino-like, as long as the decays remain prompt. The same is true for the sbottom if its decay to $b \tilde{\chi}_{1}^{0}$ is $100 \%$. An eventual improvement in these bounds, thus, would 'slide' the allowed spectrum for such a scenario to higher mass-values. When the decays are non-prompt, the bounds from LHC-8 are generally rather severe, already touching nearly a TeV.

- Depending on the relative values of $\mu_{\mathrm{eff}}, M_{2}$ and $M_{1}$, there may be situations when the lighter chargino and some other neutralinos have much varied compositions and can become light enough to appear in the cascades of the stops and the sbottoms. The combined LHC-phenomenology of such light stop and sbottom squarks may then be rather involved. Its study, however, is beyond the scope of the present work.

- The possibility of a nearly degenerate pair of relatively light stops is also demonstrated. This would strip the scenario of the interesting multi-lepton final states. On the top of that, this would alter the bounds on the stop mass since, just like $\tilde{t}_{1}, \tilde{t}_{2}$ would also cascade to $c \tilde{\chi}_{1}^{0}$ and/or to $b f \bar{f}^{\prime} \tilde{\chi}_{1}^{0}$ via an on-shell $\tilde{t}_{1}$ and a highly virtual $Z$-boson that would lead to rather soft fermion pairs escaping detection.

- We consider a rather heavy $(\gtrsim 3 \mathrm{TeV})$ gluino for our simplified, minimal setup. Enhanced contributions to the inclusive final states are foreseen for a relatively light 
gluino such that $\tilde{t}_{2}$-s could be copiously produced in its cascade. However, such yields are to be subjected to the relevant LHC constraints which, in turn, would constrain the scenario.

- We also note that the reported 1-2 $\sigma$ level disagreement in the measured $W^{+} W^{-}$ cross-section [138-140] with the SM prediction cannot be reproduced with our benchmark points. The same is true for the $\sim 3 \sigma$ dilepton excess reported by ATLAS on-Z search [141].

To summarize, a relatively light $\tilde{t}_{2}$ along with an even lighter $\tilde{t}_{1}$ is theoretically rather well-motivated but finding them together could be tricky. In the NMSSM, the situation leads to interesting possibilities at the imminent runs of the LHC experiments by having these two states active in a rather collective way. However, such a possibility is rather generic to various other new physics scenarios with extra, possibly large contributions to the mass of the SM-like Higgs boson. Thus, a dedicated strategy to find such states is called for.

\section{Acknowledgments}

JB, AC and SKR are partially supported by funding available from the Department of Atomic Energy, Government of India for the Regional Centre for Accelerator-based Particle Physics (RECAPP), Harish-Chandra Research Institute (HRI). The authors acknowledge the use of the cluster computing setup available at RECAPP and at the High Performance Computing facility of HRI. The authors would like to thank Debottam Das, Benjamin Fuks, Manoj Kumar Mandal, Olivier Mattelear, Florian Staub and Jamie Tattersall for very helpful discussions and Amit Khulve and Ravindra Yadav for technical support.

Open Access. This article is distributed under the terms of the Creative Commons Attribution License (CC-BY 4.0), which permits any use, distribution and reproduction in any medium, provided the original author(s) and source are credited.

\section{References}

[1] ATLAS collaboration, Observation of a new particle in the search for the standard model Higgs boson with the ATLAS detector at the LHC, Phys. Lett. B 716 (2012) 1 [arXiv: 1207.7214] [INSPIRE].

[2] CMS collaboration, Observation of a new boson at a mass of $125 \mathrm{GeV}$ with the CMS experiment at the LHC, Phys. Lett. B 716 (2012) 30 [arXiv:1207.7235] [INSPIRE].

[3] Z. Marshall, Searches for strongly produced SUSY at LHC, talk given at Rencontres de Moriond 2015, March 21-28, La Thuile, Italy (2015).

[4] S. Folgueras, Searches for weakly produced SUSY at LHC, talk given at Rencontres de Moriond 2015, March 21-28, La Thuile, Italy (2015).

[5] R. Barbieri and A. Strumia, The 'LEP paradox', hep-ph/0007265.

[6] U. Ellwanger, C. Hugonie and A.M. Teixeira, The next-to-minimal supersymmetric standard model, Phys. Rept. 496 (2010) 1 [arXiv:0910.1785] [INSPIRE]. 
[7] S. Chang, P.J. Fox and N. Weiner, Naturalness and Higgs decays in the MSSM with a singlet, JHEP 08 (2006) 068 [hep-ph/0511250] [INSPIRE].

[8] R. Dermisek and J.F. Gunion, A comparison of mixed-Higgs scenarios in the NMSSM and the MSSM, Phys. Rev. D 77 (2008) 015013 [arXiv:0709.2269] [InSPIRE].

[9] Z. Kang, J. Li and T. Li, On naturalness of the MSSM and NMSSM, JHEP 11 (2012) 024 [arXiv:1201.5305] [INSPIRE].

[10] J.-J. Cao, Z.-X. Heng, J.M. Yang, Y.-M. Zhang and J.-Y. Zhu, A SM-like Higgs near $125 \mathrm{GeV}$ in low energy SUSY: a comparative study for MSSM and NMSSM, JHEP 03 (2012) 086 [arXiv:1202.5821] [InSPIRE].

[11] K. Agashe, Y. Cui and R. Franceschini, Natural Islands for a $125 \mathrm{GeV}$ Higgs in the scale-invariant NMSSM, JHEP 02 (2013) 031 [arXiv:1209.2115] [INSPIRE].

[12] D. Curtin, P. Meade and P.-J. Tien, Natural SUSY in plain sight, Phys. Rev. D 90 (2014) 115012 [arXiv: 1406.0848] [INSPIRE].

[13] J.E. Kim and H.P. Nilles, The $\mu$ problem and the strong CP problem, Phys. Lett. B 138 (1984) 150 [INSPIRE].

[14] O. Stal and G. Weiglein, Light NMSSM Higgs bosons in SUSY cascade decays at the LHC, JHEP 01 (2012) 071 [arXiv:1108.0595] [INSPIRE].

[15] J.F. Gunion, Y. Jiang and S. Kraml, The constrained NMSSM and Higgs near $125 \mathrm{GeV}$, Phys. Lett. B 710 (2012) 454 [arXiv:1201.0982] [INSPIRE].

[16] S.F. King, M. Mühlleitner and R. Nevzorov, NMSSM Higgs benchmarks near $125 \mathrm{GeV}$, Nucl. Phys. B 860 (2012) 207 [arXiv:1201.2671] [INSPIRE].

[17] J.F. Gunion, Y. Jiang and S. Kraml, Could two NMSSM Higgs bosons be present near 125 GeV?, Phys. Rev. D 86 (2012) 071702 [arXiv:1207.1545] [InSPIRE].

[18] S.F. King, M. Mühlleitner, R. Nevzorov and K. Walz, Natural NMSSM Higgs bosons, Nucl. Phys. B 870 (2013) 323 [arXiv:1211.5074] [INSPIRE].

[19] J. Cao, F. Ding, C. Han, J.M. Yang and J. Zhu, A light Higgs scalar in the NMSSM confronted with the latest LHC Higgs data, JHEP 11 (2013) 018 [arXiv:1309.4939] [INSPIRE].

[20] S.F. King, M. Mühlleitner, R. Nevzorov and K. Walz, Discovery prospects for NMSSM Higgs bosons at the high-energy Large Hadron Collider, Phys. Rev. D 90 (2014) 095014 [arXiv:1408.1120] [INSPIRE].

[21] U. Ellwanger and C. Hugonie, Neutralino cascades in the $(M+1) S S M$, Eur. Phys. J. C 5 (1998) 723 [hep-ph/9712300] [inSPIRE].

[22] U. Ellwanger and C. Hugonie, Topologies of the (M+1)SSM with a singlino LSP at LEP-2, Eur. Phys. J. C 13 (2000) 681 [hep-ph/9812427] [INSPIRE].

[23] A. Dedes, C. Hugonie, S. Moretti and K. Tamvakis, Phenomenology of a new minimal supersymmetric extension of the standard model, Phys. Rev. D 63 (2001) 055009 [hep-ph/0009125] [INSPIRE].

[24] S.Y. Choi, D.J. Miller and P.M. Zerwas, The neutralino sector of the next-to-minimal supersymmetric standard model, Nucl. Phys. B 711 (2005) 83 [hep-ph/0407209] [inSPIRE]. 
[25] K. Cheung and T.-J. Hou, Light pseudoscalar Higgs boson in neutralino decays in the next-to-minimal supersymmetric standard model, Phys. Lett. B 674 (2009) 54 [arXiv:0809.1122] [INSPIRE].

[26] D.G. Cerdeño, P. Ghosh, C.B. Park and M. Peiró, Collider signatures of a light NMSSM pseudoscalar in neutralino decays in the light of LHC results, JHEP 02 (2014) 048 [arXiv:1307.7601] [INSPIRE].

[27] D. Das, U. Ellwanger and A.M. Teixeira, Modified signals for supersymmetry in the NMSSM with a singlino-like LSP, JHEP 04 (2012) 067 [arXiv:1202.5244] [INSPIRE].

[28] D. Das, U. Ellwanger and A.M. Teixeira, LHC constraints on $M_{1 / 2}$ and $m_{0}$ in the semi-constrained NMSSM, JHEP 04 (2013) 117 [arXiv:1301.7584] [INSPIRE].

[29] S. Kraml, A.R. Raklev and M.J. White, NMSSM in disguise: discovering singlino dark matter with soft leptons at the LHC, Phys. Lett. B 672 (2009) 361 [arXiv:0811.0011] [INSPIRE].

[30] G. Panotopoulos, The degenerate scenario in the NMSSM: direct singlino-like neutralino searches with a gravitino LSP, arXiv:1103.0140.

[31] U. Ellwanger, Testing the higgsino-singlino sector of the NMSSM with trileptons at the LHC, JHEP 11 (2013) 108 [arXiv:1309.1665] [INSPIRE].

[32] U. Ellwanger and A.M. Teixeira, NMSSM with a singlino LSP: possible challenges for searches for supersymmetry at the LHC, JHEP 10 (2014) 113 [arXiv:1406.7221] [INSPIRE].

[33] X.-J. Bi, Q.-S. Yan and P.-F. Yin, Light stop/sbottom pair production searches in the NMSSM, Phys. Rev. D 87 (2013) 035007 [arXiv: 1209.2703] [InSPIRE].

[34] J. Guo, Z. Kang, J. Li and T. Li, Implications of Higgs sterility for the Higgs and stop sectors, arXiv:1308.3075 [INSPIRE].

[35] A. Chakraborty, D.K. Ghosh, S. Mondal, S. Poddar and D. Sengupta, Probing the NMSSM via Higgs boson signatures from stop cascade decays at the LHC, Phys. Rev. D 91 (2015) 115018 [arXiv: 1503.07592] [INSPIRE].

[36] T. Cheng, J. Li, T. Li and Q.-S. Yan, Natural NMSSM confronting with the LHC7-8, Phys. Rev. D 89 (2014) 015015 [arXiv: 1304.3182] [INSPIRE].

[37] ATLAS, CMS collaboration, M. Flechl, Higgs physics: review of recent results and prospects from ATLAS and CMS, J. Phys. Conf. Ser. 631 (2015) 012028 [arXiv: 1503.00632] [INSPIRE].

[38] U. Ellwanger, Higgs bosons in the next-to-minimal supersymmetric standard model at the LHC, Eur. Phys. J. C 71 (2011) 1782 [arXiv:1108.0157] [InSPIRE].

[39] R. Barbieri, L.J. Hall, Y. Nomura and V.S. Rychkov, Supersymmetry without a light Higgs boson, Phys. Rev. D 75 (2007) 035007 [hep-ph/0607332] [INSPIRE].

[40] R. Barbieri, L.J. Hall, A.Y. Papaioannou, D. Pappadopulo and V.S. Rychkov, An alternative NMSSM phenomenology with manifest perturbative unification, JHEP 03 (2008) 005 [arXiv:0712.2903] [INSPIRE].

[41] Y. Okada, M. Yamaguchi and T. Yanagida, Upper bound of the lightest Higgs boson mass in the minimal supersymmetric standard model, Prog. Theor. Phys. 85 (1991) 1 [INSPIRE].

[42] J.R. Ellis, G. Ridolfi and F. Zwirner, Radiative corrections to the masses of supersymmetric Higgs bosons, Phys. Lett. B 257 (1991) 83 [InSPIRE]. 
[43] H.E. Haber and R. Hempfling, Can the mass of the lightest Higgs boson of the minimal supersymmetric model be larger than $m(Z)$ ?, Phys. Rev. Lett. 66 (1991) 1815 [INSPIRE].

[44] M. Carena, J.R. Espinosa, M. Quirós and C.E.M. Wagner, Analytical expressions for radiatively corrected Higgs masses and couplings in the MSSM, Phys. Lett. B 355 (1995) 209 [hep-ph/9504316] [INSPIRE].

[45] M. Carena, M. Quirós and C.E.M. Wagner, Effective potential methods and the Higgs mass spectrum in the MSSM, Nucl. Phys. B 461 (1996) 407 [hep-ph/9508343] [INSPIRE].

[46] H.E. Haber, R. Hempfling and A.H. Hoang, Approximating the radiatively corrected Higgs mass in the minimal supersymmetric model, Z. Phys. C 75 (1997) 539 [hep-ph/9609331] [INSPIRE].

[47] A. Djouadi, The Anatomy of electro-weak symmetry breaking. II. The Higgs bosons in the minimal supersymmetric model, Phys. Rept. 459 (2008) 1 [hep-ph/0503173] [INSPIRE].

[48] A. Arbey, M. Battaglia, A. Djouadi, F. Mahmoudi and J. Quevillon, Implications of a 125 GeV Higgs for supersymmetric models, Phys. Lett. B 708 (2012) 162 [arXiv:1112.3028] [INSPIRE].

[49] P. Draper, P. Meade, M. Reece and D. Shih, Implications of a $125 \mathrm{GeV}$ Higgs for the MSSM and low-scale SUSY breaking, Phys. Rev. D 85 (2012) 095007 [arXiv:1112.3068] [InSPIRE].

[50] M. Carena, S. Gori, N.R. Shah and C.E.M. Wagner, A $125 \mathrm{GeV}$ SM-like Higgs in the MSSM and the $\gamma \gamma$ rate, JHEP 03 (2012) 014 [arXiv:1112.3336] [INSPIRE].

[51] D. Carmi, A. Falkowski, E. Kuflik and T. Volansky, Interpreting LHC Higgs results from natural new physics perspective, JHEP 07 (2012) 136 [arXiv:1202.3144] [INSPIRE].

[52] A. Arbey, M. Battaglia, A. Djouadi and F. Mahmoudi, The Higgs sector of the phenomenological MSSM in the light of the Higgs boson discovery, JHEP 09 (2012) 107 [arXiv: 1207.1348] [INSPIRE].

[53] P. Bechtle, S. Heinemeyer, O. Stal, T. Stefaniak, G. Weiglein and L. Zeune, MSSM interpretations of the LHC discovery: light or heavy Higgs?, Eur. Phys. J. C $\mathbf{7 3}$ (2013) 2354 [arXiv: 1211.1955] [INSPIRE].

[54] U. Ellwanger and C. Hugonie, The upper bound on the lightest Higgs mass in the NMSSM revisited, Mod. Phys. Lett. A 22 (2007) 1581 [hep-ph/0612133] [INSPIRE].

[55] L.J. Hall, D. Pinner and J.T. Ruderman, A natural SUSY Higgs near 126 GeV, JHEP 04 (2012) 131 [arXiv:1112.2703] [INSPIRE].

[56] A. Arvanitaki and G. Villadoro, A non standard model Higgs at the LHC as a sign of naturalness, JHEP 02 (2012) 144 [arXiv:1112.4835] [INSPIRE].

[57] M. Papucci, J.T. Ruderman and A. Weiler, Natural SUSY endures, JHEP 09 (2012) 035 [arXiv:1110.6926] [INSPIRE].

[58] U. Ellwanger, J.F. Gunion and C. Hugonie, NMHDECAY: a Fortran code for the Higgs masses, couplings and decay widths in the NMSSM, JHEP 02 (2005) 066 [hep-ph/0406215] [INSPIRE].

[59] U. Ellwanger and C. Hugonie, NMHDECAY 2.0: an updated program for sparticle masses, Higgs masses, couplings and decay widths in the NMSSM, Comput. Phys. Commun. 175 (2006) 290 [hep-ph/0508022] [INSPIRE]. 
[60] U. Ellwanger and C. Hugonie, NMSPEC: a Fortran code for the sparticle and Higgs masses in the NMSSM with GUT scale boundary conditions, Comput. Phys. Commun. 177 (2007) 399 [hep-ph/0612134] [INSPIRE].

[61] U. Ellwanger, G. Espitalier-Noel and C. Hugonie, Naturalness and fine tuning in the NMSSM: implications of early LHC results, JHEP 09 (2011) 105 [arXiv:1107.2472] [INSPIRE].

[62] D. Das, U. Ellwanger and A.M. Teixeira, NMSDECAY: a Fortran code for supersymmetric particle decays in the next-to-minimal supersymmetric standard model, Comput. Phys. Commun. 183 (2012) 774 [arXiv:1106.5633] [INSPIRE].

[63] M. Muhlleitner, A. Djouadi and Y. Mambrini, SDECAY: a Fortran code for the decays of the supersymmetric particles in the MSSM, Comput. Phys. Commun. 168 (2005) 46 [hep-ph/0311167] [INSPIRE].

[64] M. Carena, S. Heinemeyer, O. Stål, C.E.M. Wagner and G. Weiglein, MSSM Higgs boson searches at the LHC: benchmark scenarios after the discovery of a Higgs-like particle, Eur. Phys. J. C 73 (2013) 2552 [arXiv: 1302.7033] [INSPIRE].

[65] ATLAS, CMS collaboration, Combined measurement of the Higgs boson mass in pp collisions at $\sqrt{s}=7$ and $8 \mathrm{TeV}$ with the ATLAS and CMS experiments, Phys. Rev. Lett. 114 (2015) 191803 [arXiv: 1503.07589] [INSPIRE].

[66] M.D. Goodsell, K. Nickel and F. Staub, Two-loop corrections to the Higgs masses in the NMSSM, Phys. Rev. D 91 (2015) 035021 [arXiv:1411.4665] [InSPIRE].

[67] S. Heinemeyer, W. Hollik and G. Weiglein, FeynHiggs: a program for the calculation of the masses of the neutral CP even Higgs bosons in the MSSM, Comput. Phys. Commun. 124 (2000) 76 [hep-ph/9812320] [INSPIRE].

[68] S. Heinemeyer, W. Hollik and G. Weiglein, The masses of the neutral CP-even Higgs bosons in the MSSM: Accurate analysis at the two loop level, Eur. Phys. J. C 9 (1999) 343 [hep-ph/9812472] [INSPIRE].

[69] G. Degrassi, S. Heinemeyer, W. Hollik, P. Slavich and G. Weiglein, Towards high precision predictions for the MSSM Higgs sector, Eur. Phys. J. C 28 (2003) 133 [hep-ph/0212020] [INSPIRE].

[70] M. Frank, T. Hahn, S. Heinemeyer, W. Hollik, H. Rzehak and G. Weiglein, The Higgs boson masses and mixings of the complex MSSM in the Feynman-diagrammatic approach, JHEP 02 (2007) 047 [hep-ph/0611326] [INSPIRE].

[71] T. Hahn, S. Heinemeyer, W. Hollik, H. Rzehak and G. Weiglein, High-precision predictions for the light CP-even Higgs boson mass of the minimal supersymmetric standard model, Phys. Rev. Lett. 112 (2014) 141801 [arXiv:1312.4937] [INSPIRE].

[72] A. Djouadi, J.-L. Kneur and G. Moultaka, SuSpect: a Fortran code for the supersymmetric and Higgs particle spectrum in the MSSM, Comput. Phys. Commun. 176 (2007) 426 [hep-ph/0211331] [INSPIRE].

[73] N. Blinov and D.E. Morrissey, Charge and color breaking constraints in the minimal supersymmetric standard model, arXiv:1309.7397.

[74] D. Chowdhury, R.M. Godbole, K.A. Mohan and S.K. Vempati, Charge and color breaking constraints in MSSM after the Higgs discovery at LHC, JHEP 02 (2014) 110 [arXiv:1310.1932] [INSPIRE]. 
[75] U. Chattopadhyay and A. Dey, Exploring MSSM for charge and color breaking and other constraints in the context of Higgs@125 GeV, JHEP 11 (2014) 161 [arXiv:1409.0611] [INSPIRE].

[76] J.E. Camargo-Molina, B. O'Leary, W. Porod and F. Staub, Stability of the CMSSM against sfermion VEVs, JHEP 12 (2013) 103 [arXiv:1309.7212] [INSPIRE].

[77] J.E. Camargo-Molina, B. Garbrecht, B. O'Leary, W. Porod and F. Staub, Constraining the natural MSSM through tunneling to color-breaking vacua at zero and non-zero temperature, Phys. Lett. B 737 (2014) 156 [arXiv:1405.7376] [inSPIRE].

[78] N. Blinov and D.E. Morrissey, Vacuum stability and the MSSM Higgs mass, JHEP 03 (2014) 106 [arXiv: 1310.4174] [INSPIRE].

[79] M. Bobrowski, G. Chalons, W.G. Hollik and U. Nierste, Vacuum stability of the effective Higgs potential in the Minimal Supersymmetric Standard Model, Phys. Rev. D 90 (2014) 035025 [arXiv: 1407.2814] [INSPIRE].

[80] K.-i. Hikasa and M. Kobayashi, Light scalar top at $e^{+} e^{-}$colliders, Phys. Rev. D 36 (1987) 724 [INSPIRE].

[81] M. Muhlleitner and E. Popenda, Light stop decay in the MSSM with minimal flavour violation, JHEP 04 (2011) 095 [arXiv: 1102.5712] [INSPIRE].

[82] C. Boehm, A. Djouadi and Y. Mambrini, Decays of the lightest top squark, Phys. Rev. D 61 (2000) 095006 [hep-ph/9907428] [INSPIRE].

[83] S.P. Das, A. Datta and M. Guchait, Four-body decay of the stop squark at the upgraded Tevatron, Phys. Rev. D 65 (2002) 095006 [hep-ph/0112182] [INSPIRE].

[84] ATLAS collaboration, Search for direct top squark pair production in events with a $Z$ boson, b-jets and missing transverse momentum in $\sqrt{s}=8 \mathrm{TeV}$ pp collisions with the ATLAS detector, Eur. Phys. J. C 74 (2014) 2883 [arXiv:1403.5222] [InSPIRE].

[85] CMS collaboration, Search for top-squark pairs decaying into Higgs or $Z$ bosons in pp collisions at $\sqrt{s}=8 \mathrm{TeV}$, Phys. Lett. B 736 (2014) 371 [arXiv:1405.3886] [INSPIRE].

[86] D. Ghosh, Boosted dibosons from mixed heavy top squarks, Phys. Rev. D 88 (2013) 115013 [arXiv: 1308.0320] [INSPIRE].

[87] ATLAS collaboration, Search for direct third-generation squark pair production in final states with missing transverse momentum and two b-jets in $\sqrt{s}=8 \mathrm{TeV}$ pp collisions with the ATLAS detector, JHEP 10 (2013) 189 [arXiv:1308.2631] [INSPIRE].

[88] CMS collaboration, Search for direct production of bottom squark pairs, CMS-PAS-SUS-14-011 (2014).

[89] ATLAS collaboration, Search for pair-produced third-generation squarks decaying via charm quarks or in compressed supersymmetric scenarios in pp collisions at $\sqrt{s}=8 \mathrm{TeV}$ with the ATLAS detector, Phys. Rev. D 90 (2014) 052008 [arXiv:1407.0608] [INSPIRE].

[90] CMS collaboration, Search for top squark decaying to a charm quark and a neutralino in events with a jet and missing transverse momentum, CMS-PAS-SUS-13-009 (2013).

[91] ATLAS collaboration, Search for top squark pair production in final states with one isolated lepton, jets and missing transverse momentum in $\sqrt{s}=8 \mathrm{TeV}$ pp collisions with the ATLAS detector, JHEP 11 (2014) 118 [arXiv:1407.0583] [INSPIRE]. 
[92] H. Li, W. Parker, Z. Si and S. Su, Sbottom signature of the supersymmetric golden region, Eur. Phys. J. C 71 (2011) 1584 [arXiv: 1009.6042] [INSPIRE].

[93] A. Datta and S. Niyogi, Entangled system of squarks from the third generation at the Large Hadron Collider, arXiv:1111.0200 [INSPIRE].

[94] G. Bélanger, F. Boudjema, C. Hugonie, A. Pukhov and A. Semenov, Relic density of dark matter in the NMSSM, JCAP 09 (2005) 001 [hep-ph/0505142] [INSPIRE].

[95] Muon G-2 collaboration, G.W. Bennett et al., Final report of the muon E821 anomalous magnetic moment measurement at BNL, Phys. Rev. D 73 (2006) 072003 [hep-ex/0602035] [INSPIRE].

[96] T. Blum et al., The muon $(g-2)$ theory value: present and future, arXiv:1311.2198 [INSPIRE].

[97] M. Endo, K. Hamaguchi, S. Iwamoto and T. Yoshinaga, Muon g- 2 vs LHC in supersymmetric models, JHEP 01 (2014) 123 [arXiv:1303.4256] [INSPIRE].

[98] M. Badziak, Z. Lalak, M. Lewicki, M. Olechowski and S. Pokorski, Upper bounds on sparticle masses from muon $g-2$ and the Higgs mass and the complementarity of future colliders, JHEP 03 (2015) 003 [arXiv:1411.1450] [INSPIRE].

[99] Planck collaboration, P.A.R. Ade et al., Planck 2015 results. XIII. Cosmological parameters, arXiv:1502.01589 [INSPIRE].

[100] LUX collaboration, D.S. Akerib et al., First results from the LUX dark matter experiment at the Sanford Underground Research Facility, Phys. Rev. Lett. 112 (2014) 091303 [arXiv: 1310 . 8214] [INSPIRE].

[101] T. Moroi and L. Randall, Wino cold dark matter from anomaly mediated SUSY breaking, Nucl. Phys. B 570 (2000) 455 [hep-ph/9906527] [INSPIRE].

[102] B.S. Acharya, P. Kumar, K. Bobkov, G. Kane, J. Shao and S. Watson, Non-thermal dark matter and the moduli problem in string frameworks, JHEP 06 (2008) 064 [arXiv:0804.0863] [INSPIRE].

[103] B.S. Acharya, G. Kane, S. Watson and P. Kumar, A non-thermal WIMP miracle, Phys. Rev. D 80 (2009) 083529 [arXiv: 0908.2430] [InSPIRE].

[104] E. Hardy, J. March-Russell and J. Unwin, Precision unification in $\lambda S U S Y$ with a $125 \mathrm{GeV}$ Higgs, JHEP 10 (2012) 072 [arXiv:1207.1435] [INSPIRE].

[105] M. Farina, M. Perelstein and B. Shakya, Higgs couplings and naturalness in $\lambda$-SUSY, JHEP 04 (2014) 108 [arXiv: 1310.0459] [INSPIRE].

[106] M. Drees, H. Dreiner, D. Schmeier, J. Tattersall and J.S. Kim, CheckMATE: confronting your favourite new physics model with LHC data, Comput. Phys. Commun. 187 (2014) 227 [arXiv:1312.2591] [INSPIRE].

[107] K. Krizka, A. Kumar and D.E. Morrissey, Very light scalar top quarks at the LHC, Phys. Rev. D 87 (2013) 095016 [arXiv:1212.4856] [INSPIRE].

[108] A. Delgado, G.F. Giudice, G. Isidori, M. Pierini and A. Strumia, The light stop window, Eur. Phys. J. C 73 (2013) 2370 [arXiv:1212.6847] [INSPIRE].

[109] G. Bélanger, D. Ghosh, R. Godbole, M. Guchait and D. Sengupta, Probing the flavor violating scalar top quark signal at the LHC, Phys. Rev. D 89 (2014) 015003 [arXiv: 1308.6484] [INSPIRE]. 
[110] R. Grober, M. Muhlleitner, E. Popenda and A. Wlotzka, Light stop decays: implications for LHC searches, arXiv: 1408.4662 [INSPIRE].

[111] G. Ferretti, R. Franceschini, C. Petersson and R. Torre, Spot the stop with a b-tag, Phys. Rev. Lett. 114 (2015) 201801 [arXiv:1502.01721] [INSPIRE].

[112] B. Dutta et al., Probing compressed top squark scenarios at the LHC at $14 \mathrm{TeV}$, Phys. Rev. D 90 (2014) 095022 [arXiv:1312.1348] [INSPIRE].

[113] W.S. Cho et al., Improving the sensitivity of stop searches with on-shell constrained invariant mass variables, JHEP 05 (2015) 040 [arXiv:1411.0664] [INSPIRE].

[114] J. Eckel, S. Su and H. Zhang, Complex decay chains of top and bottom squarks, JHEP 07 (2015) 075 [arXiv: 1411.1061] [InSPIRE].

[115] L. Covi and F. Dradi, Long-Lived stop at the LHC with or without R-parity, JCAP 10 (2014) 039 [arXiv: 1403.4923] [INSPIRE].

[116] ATLAS collaboration, Searches for heavy long-lived charged particles with the ATLAS detector in proton-proton collisions at $\sqrt{s}=8 \mathrm{TeV}$, JHEP 01 (2015) 068 [arXiv: 1411.6795] [INSPIRE].

[117] A. de la Puente and A. Szynkman, Long-lived Colored Scalars at the LHC, arXiv: 1504.07293 [INSPIRE].

[118] J. Alwall et al., The automated computation of tree-level and next-to-leading order differential cross sections and their matching to parton shower simulations, JHEP $\mathbf{0 7}$ (2014) 079 [arXiv : 1405.0301] [inSPIRE].

[119] A. Belyaev, N.D. Christensen and A. Pukhov, CalcHEP 3.4 for collider physics within and beyond the Standard Model, Comput. Phys. Commun. 184 (2013) 1729 [arXiv:1207.6082] [INSPIRE].

[120] ATLAS collaboration, Calibration of the performance of b-tagging for $c$ and light-flavour jets in the 2012 ATLAS data, ATLAS-CONF-2014-046 (2014).

[121] ATLAS collaboration, Performance and calibration of the JetFitterCharm algorithm for c-jet identification, ATL-PHYS-PUB-2015-001 (2015).

[122] J. Pumplin, D.R. Stump, J. Huston, H.L. Lai, P.M. Nadolsky and W.K. Tung, New generation of parton distributions with uncertainties from global QCD analysis, JHEP 07 (2002) 012 [hep-ph/0201195] [INSPIRE].

[123] W. Beenakker, M. Krämer, T. Plehn, M. Spira and P.M. Zerwas, Stop production at hadron colliders, Nucl. Phys. B 515 (1998) 3 [hep-ph/9710451] [InSPIRE].

[124] http://www.thphys.uni-heidelberg.de/ plehn/index.php?show=prospino\&visible=tools

[125] T. Sjöstrand, S. Mrenna and P.Z. Skands, PYTHIA 6.4 physics and manual, JHEP 05 (2006) 026 [hep-ph/0603175] [INSPIRE].

[126] B.C. Allanach et al., SUSY Les Houches accord 2, Comput. Phys. Commun. 180 (2009) 8 [arXiv:0801.0045] [INSPIRE].

[127] M.L. Mangano, M. Moretti, F. Piccinini and M. Treccani, Matching matrix elements and shower evolution for top-quark production in hadronic collisions, JHEP 01 (2007) 013 [hep-ph/0611129] [INSPIRE].

[128] M.L. Mangano, The so-called MLM prescription for ME/PS matching, presented at the Fermilab ME/MC Tuning Workshop, October 4, Fermilab, U.S.A. (2002). 
[129] J. Alwall et al., Comparative study of various algorithms for the merging of parton showers and matrix elements in hadronic collisions, Eur. Phys. J. C 53 (2008) 473 [arXiv:0706.2569] [INSPIRE].

[130] DELPHES 3 collaboration, J. de Favereau et al., DELPHES 3, a modular framework for fast simulation of a generic collider experiment, JHEP 02 (2014) 057 [arXiv:1307.6346] [INSPIRE].

[131] M. Cacciari, G.P. Salam and G. Soyez, FastJet user manual, Eur. Phys. J. C 72 (2012) 1896 [arXiv: 1111.6097] [INSPIRE].

[132] ATLAS collaboration, Search for supersymmetry at $\sqrt{s}=8 \mathrm{TeV}$ in final states with jets and two same-sign leptons or three leptons with the ATLAS detector, JHEP 06 (2014) 035 [arXiv: 1404.2500] [INSPIRE].

[133] E. Conte, B. Fuks and G. Serret, MadAnalysis 5, a user-friendly framework for collider phenomenology, Comput. Phys. Commun. 184 (2013) 222 [arXiv:1206.1599] [INSPIRE].

[134] E. Conte, B. Dumont, B. Fuks and C. Wymant, Designing and recasting LHC analyses with MadAnalysis 5, Eur. Phys. J. C 74 (2014) 3103 [arXiv:1405.3982] [InSPIRE].

[135] https://root.cern.ch/drupal/

[136] http://cepa.fnal.gov/psm/stdhep/

[137] CMS collaboration, CMS technical design report, volume II: physics performance, J. Phys. G 34 (2007) 995 [INSPIRE].

[138] ATLAS collaboration, Measurement of $W^{+} W^{-}$production in pp collisions at $\sqrt{s}=7 \mathrm{TeV}$ with the ATLAS detector and limits on anomalous $W W Z$ and $W W \gamma$ couplings, Phys. Rev. D 87 (2013) 112001 [arXiv:1210.2979] [INSPIRE].

[139] CMS collaboration, Measurement of the $W^{+} W^{-}$cross section in pp collisions at $\sqrt{s}=7$ TeV and limits on anomalous $W W \gamma$ and $W W Z$ couplings, Eur. Phys. J. C 73 (2013) 2610 [arXiv:1306.1126] [INSPIRE].

[140] CMS collaboration, Measurement of $W^{+} W^{-}$and $Z Z$ production cross sections in $p p$ collisions at $\sqrt{s}=8 \mathrm{TeV}$, Phys. Lett. B 721 (2013) 190 [arXiv:1301.4698] [InSPIRE].

[141] ATLAS collaboration, Search for supersymmetry in events containing a same-flavour opposite-sign dilepton pair, jets and large missing transverse momentum in $\sqrt{s}=8 \mathrm{TeV} \mathrm{pp}$ collisions with the ATLAS detector, Eur. Phys. J. C 75 (2015) 318 [arXiv:1503.03290] [INSPIRE]. 